\title{
The effectiveness of psychological interventions for fatigue in cancer survivors: systematic review of randomised controlled trials
}

\author{
T. K. Corbett ${ }^{1 *}$ D, A. Groarke ${ }^{2}$ D. Devane ${ }^{3}$, E. Carr², J. C. Walsh² and B. E. McGuire ${ }^{2}$
}

\begin{abstract}
Background: Fatigue is a common symptom in cancer patients that can persist beyond the curative treatment phase. This systematic review evaluated the effectiveness of psychological interventions for cancer-related fatigue in post-treatment cancer survivors.

Methods: We searched relevant online databases and sources of grey literature. Randomised controlled trials (RCTs) evaluating psychological interventions in adult cancer patients after the completion of treatment, with fatigue as an outcome measure, were included. Two review authors extracted data independently from the selected studies and assessed the methodological quality using the Cochrane Collaboration Risk of Bias Tool.

Results: Thirty-three psychological interventions were identified. The sample size of the included studies varied between 28 and 409, with 4525 participants overall. Twenty-three of the included studies reported a significant effect of the interventions on reducing fatigue in cancer survivors. Most interventions focused on psychoeducation, mindfulness, cognitive or behaviour therapy-oriented strategies. However, studies differed widely in terms of measurement tools used to assess fatigue, mode, duration and frequency of the intervention delivery.

Conclusions: This review showed some tentative support for psychological interventions for fatigue after cancer treatment. However, as the RCTs were heterogeneous in nature and the number of high-quality studies was limited, definitive conclusions are not yet possible. With the growing need for stage-specific research in cancer, this review sought to inform current practice and to summarise the existing evidence base of randomised controlled trials in the area.
\end{abstract}

Systematic review registration: PROSPERO registration number: CRD42014015219.

Keywords: Cancer, Psychological, Survivorship, Fatigue, Post-treatment, Cancer-related fatigue, Psychooncology, Review, Narrative review

\section{Highlights}

- The majority of treatments comprise standard components of CBT, mindfulness and/or psychoeducation. Studies comparing active psychological therapies are scarce. There is insufficient high-quality evidence to recommend

\footnotetext{
* Correspondence: T.k.corbett@soton.ac.uk

${ }^{1}$ NIHR ARC Wessex, School of Health Sciences, University of Southampton, Highfield, Southampton SO17 1BJ, UK
}

Full list of author information is available at the end of the article psychological treatment as having possible benefit for cancer-related fatigue in post-treatment cancer survivors. There is no reported evidence of adverse effects.

- The majority of the evidence is for the treatment of fatigue in those with breast cancer but there is insufficient evidence to indicate if the treatments are more effective for one type of cancer over another.

- The interventions appear to have had some impact on mood, self-efficacy to cope with fatigue and quality of life/functional impact of fatigue. However, 
there appeared to be little impact of the interventions on pain. Interventions designed specifically for $\mathrm{CrF}$ did not tend to assess sleep variables.

- With wide-ranging heterogeneity in study design and measures used to assess the outcomes, it is difficult to evaluate which format or elements reduce fatigue after cancer treatment. Furthermore, the optimum time to intervene after treatment has ended is not clear.

\section{Background}

Cancer-related fatigue ( $\mathrm{CrF}$ ) is commonly defined as 'a distressing, persistent, subjective sense of physical, emotional and/or cognitive tiredness or exhaustion related to cancer and/or cancer treatment that is not proportional to recent activity, and significantly interferes with usual functioning' [1]. There is little understanding of the underlying aetiology of $\mathrm{CrF}$ [2] but it is considered a multidimensional symptom that is comprised of physical, mental, and emotional aspects $[1,3,4]$.

There is limited evidence of the effectiveness of pharmacological interventions for the management of $\mathrm{CrF}$ [5]. However, some reviews of non-pharmacological interventions have indicated that psychological and activity-based interventions may be effective $[2,6]$. Interventions that incorporate restorative approaches, supportive-expressive techniques and cognitive-behavioural psychosocial interventions may reduce levels of $\mathrm{CrF}[6,7]$. In this review, we have focused on psychological therapies designed to improve functioning and/or reduce the physical and psychological impact of $\mathrm{CrF}$.

Psychological interventions such as cognitive-behavioural therapy (CBT) aim to influence or change cognitions, emotions, behaviours or a combination of these [8]. Interventions which target these processes may improve symptom management in $\mathrm{CrF}$ [9]. These therapies may increase knowledge, improve emotional adjustment and enhance quality of life, and have also been associated with improved coping skills, physical health and functional adjustment $[6,10]$. Patients and healthcare professionals have been reported to have high expectations of, and relatively positive attitudes towards, psychological therapies [10].

There is some evidence that psychosocial interventions are effective in reducing fatigue in patients undergoing active treatment for cancer [8]. While biological insults such as cancer or cancer treatment may lead to fatigue symptoms during the treatment phase of those with cancer, behavioural and cognitive variables may prolong fatigue during to posttreatment phase [1]. However, it is still unclear whether psychological interventions are helpful for managing fatigue in post-treatment cancer survivors beyond the early diagnostic and treatment phase [11]. Consequently, there is a need to conduct a critical review of the literature pertaining to psychological interventions in post-treatment cancer survivorship.

\section{Objectives}

This review systematically reviews and synthesizes the evidence from randomised controlled trials (RCTs) investigating the effectiveness of psychological interventions for persistent fatigue in people after the completion of cancer treatment.

\section{Methods}

The review protocol was registered with the International Prospective Register of Systematic Reviews (PROSPERO) database (registration number: CRD42014015219) and the protocol has been published [12]. The review is reported in accordance with the Preferred Reporting Items for Systematic Reviews and Meta-Analyses (PRISMA) statement [13].

\section{Criteria for considering studies for this review Types of studies}

RCTs comparing psychological treatments with no intervention (i.e. usual care or wait list controls), attention controls or another intervention for CrF. Studies were included regardless of treatment intensity or duration, mode of treatment delivery (e.g. individual, group) or medium of treatment (e.g. in-person, online). We did not impose date restrictions. Studies found in the grey literature were included if a full-text paper in English was available, either through databases or through contact with the study authors.

\section{Types of participants}

Adults 18 years and older who had completed treatment for cancer regardless of gender, tumour type, and type of medical treatment received.

\section{Types of interventions}

We included studies that evaluated the effect of psychological therapies in the management of $\mathrm{CrF}$. Interventions including psychotherapy and psycho-education were included. These interventions included those that provided advice or information (verbal, written, audio-visual or computer delivered material) in order to help people understand and manage $\mathrm{CrF}$, strategies such as cognitive restructuring, coping skill development, meditation or relaxation techniques. Studies that combined psychobehavioural and non-psychological methods were included only if the study had a predominant emphasis on a psychological element in the design. Studies were excluded if they did not employ a psychotherapeutic rationale or theory in the intervention design [12]. 


\section{Types of outcome measures}

Studies were required to have 'fatigue' as an outcome of interest. In line with Goedendorp et al. [8], studies were included if fatigue was measured with a questionnaire designed specifically to evaluate fatigue. Fatigue subscales that were part of a broader quality-of-life measure were also included, if specific fatigue-related data were available. Fatigue could also be measured with a visual analogue scale (VAS) or as part of a symptom list and scored as 'present' or 'absent'. Fatigue could be measured in terms of characteristics such as intensity, distress, duration, frequency, or as dimensions such as physical fatigue, mental fatigue or general fatigue.

\section{Secondary outcomes included}

- Functional impact of fatigue (self-report questionnaires measures assessing the impact of fatigue on daily functioning)

- Fatigue self-efficacy (self-reported scales of control or self-efficacy in relation to fatigue)

- Mood (self-reported scales of depression, and/or anxiety, or distress)

- Global quality of life (self-report questionnaires measures assessing the impact of fatigue on quality of life).

\section{Information sources}

The following electronic databases were searched: Cochrane Central Register of Controlled Trials (CENTRAL), MEDLINE, EMBASE, CINAHL PsycINFO, Web of Science and CancerLit. Alterations were made to the search strategies as appropriate for each database. An example search strategy can be seen in Table 1 (See Additional file 3. For further details of the search strategies used). The original search was conducted on October 6th and 7th 2015 and was updated on the 22nd and 23rd of January 2018. Studies from 2014 to 2018 were assessed for inclusion based on the criteria followed in the original search.

Unpublished and ongoing trials were identified by checking appropriate databases of current ongoing clinical research studies. Grey literature was searched using the
OpenGrey database (www.opengrey.eu), which includes technical or research reports or doctoral dissertations. Conference papers from annual American Society of Clinical Oncology (ASCO) or International Psycho Oncology Society World Congress (IPOS) conferences were also searched. Other published, unpublished and ongoing trials were identified by checking trials and protocols published on the following clinical trials registers and websites.

- World Health Organization International Clinical Trials Registry Platform (WHO ICTRP; www.who.int/ ictrp/en).

- metaRegister of Controlled Trials (mRCT; www. controlled-trials.com $/ \mathrm{mrct} /$ ).

- ClinicalTrials.gov (www.clinicaltrials.gov).

- www.cancer.gov/clinicaltrials.

Search methods for identification of studies

\section{Data collection and analysis}

One review author (TC) conducted the initial search before screening titles. Titles that were clearly not relevant to this review were removed. Three review authors (TC, $\mathrm{EC}$ and $\mathrm{BMG}$ ) independently screened the remaining titles and abstracts for their eligibility for inclusion. Ineligible studies were excluded at this stage, with each author recording the reason for rejection. Full-text copies were retrieved and screened if the title and abstract did not provide sufficient information concerning the inclusion criteria for this review. Copies of all studies that possibly or definitely met the inclusion criteria were also retrieved. Disagreements between the reviewers were resolved by discussion, with the involvement of another reviewer where agreement could not be reached (DD). Multiple reports of the same study were included as a single study, with each study identified by the lead author of the primary results paper.

\section{Data extraction and management}

Review authors (TC, EC, AG and BMG) extracted data independently from the studies using a specifically designed data extraction form (see Table 2). Authors were contacted where further clarity regarding the study was required, or in order to obtain additional data.

Table 1 Sample search strategy: details of the terms searched in CINAHL database

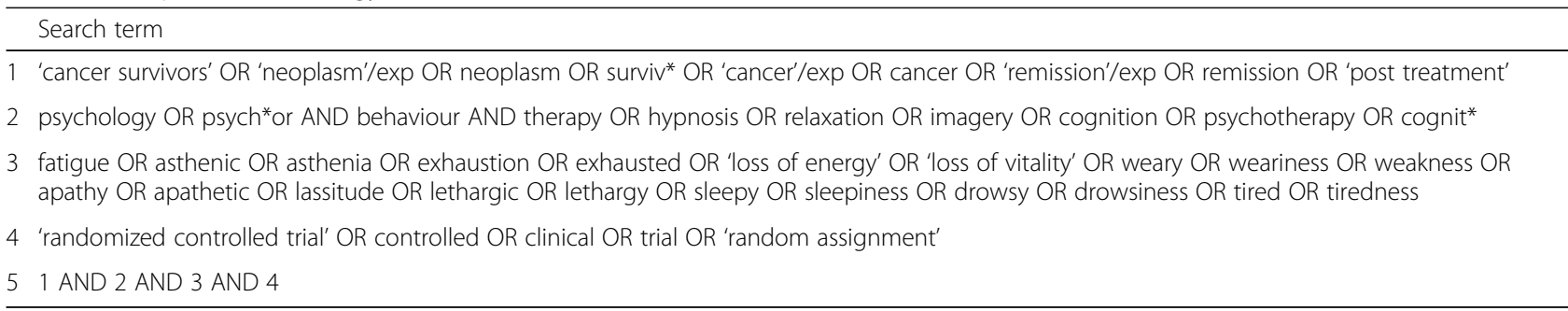




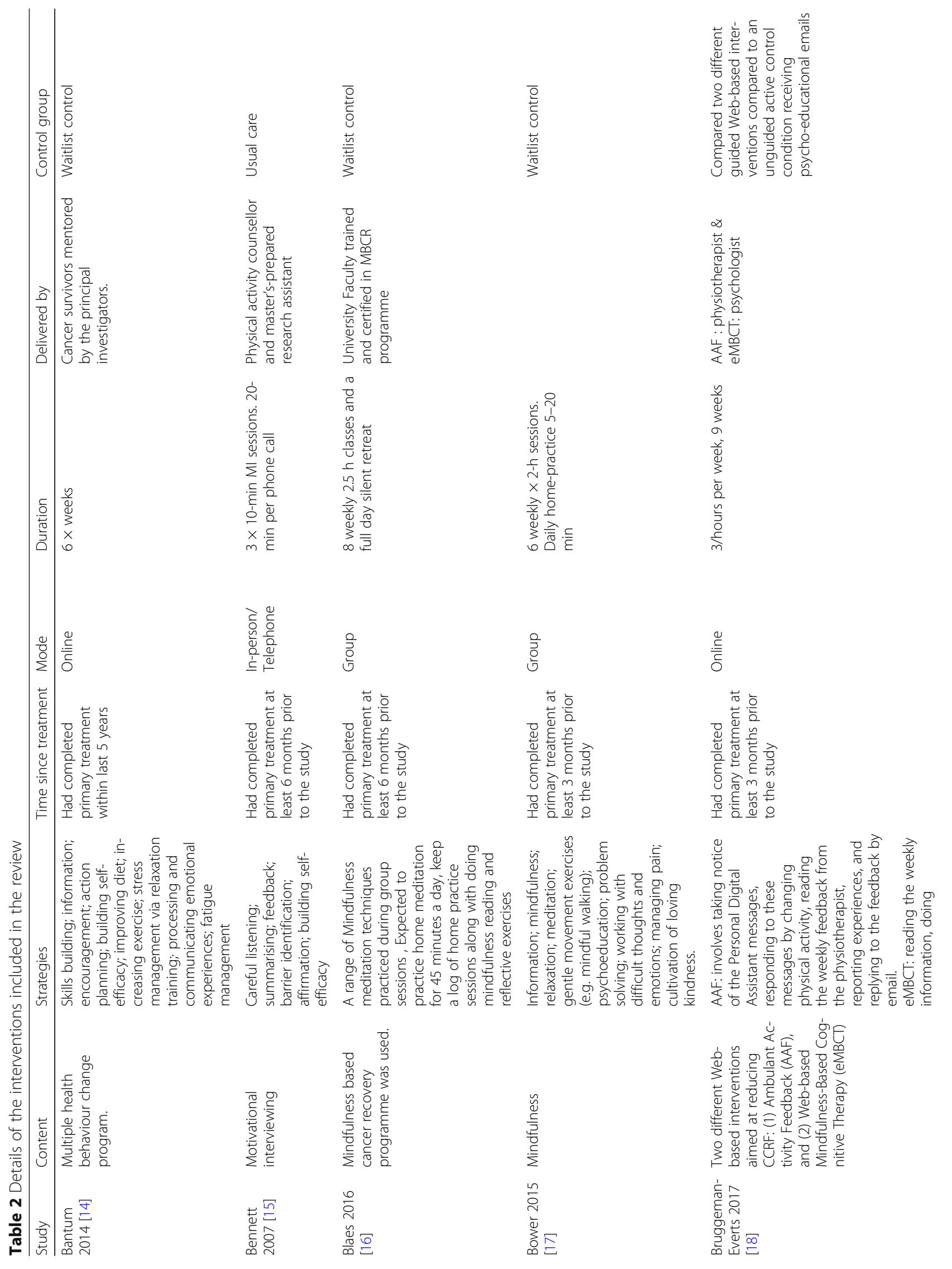




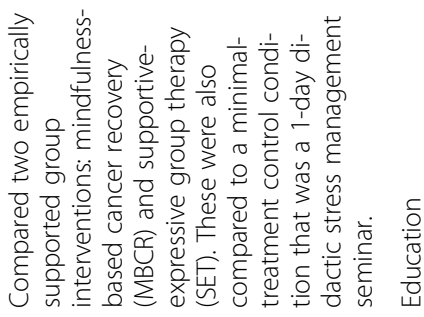

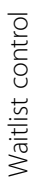
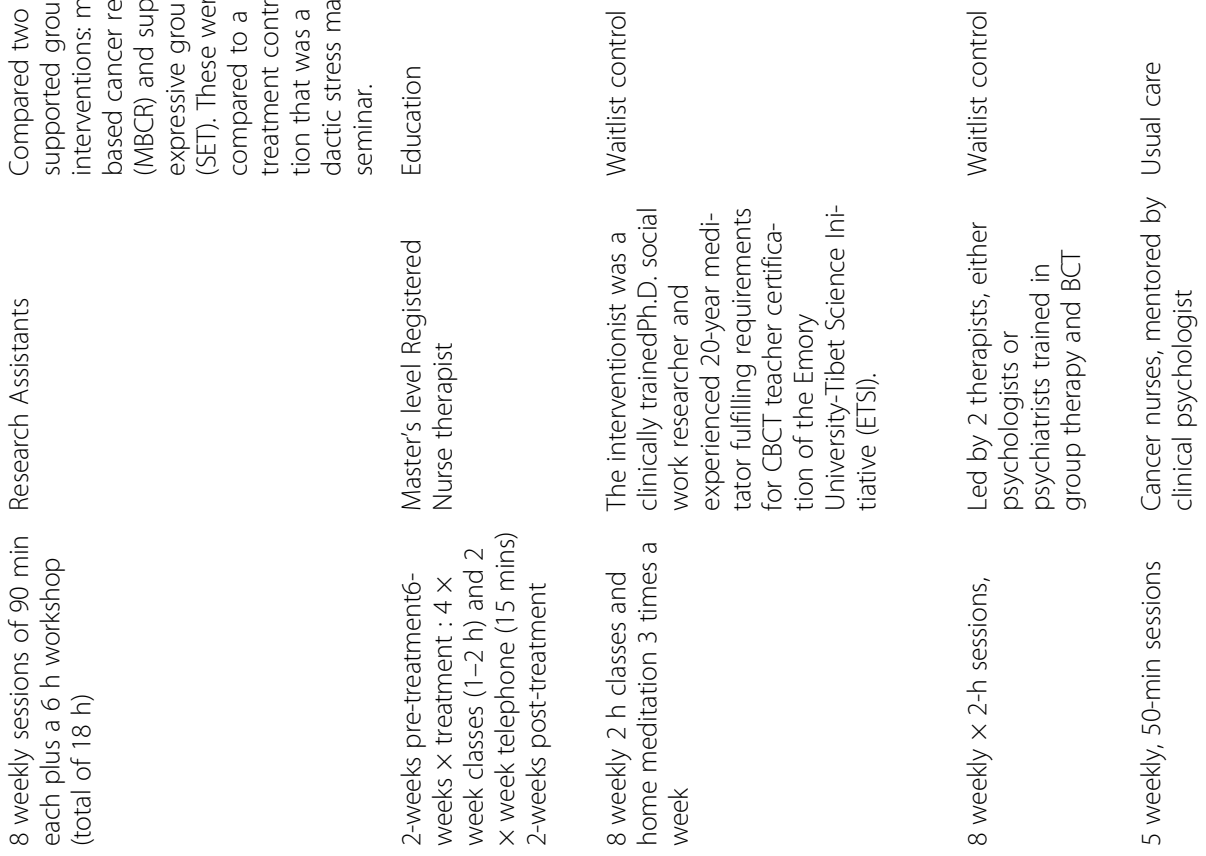

인

$\frac{0}{\frac{0}{0}}$

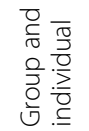

$\frac{\circ}{\frac{0}{0}}$<smiles>COCCOCOC</smiles>

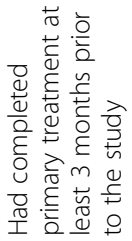

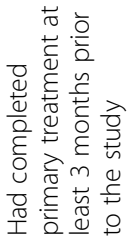
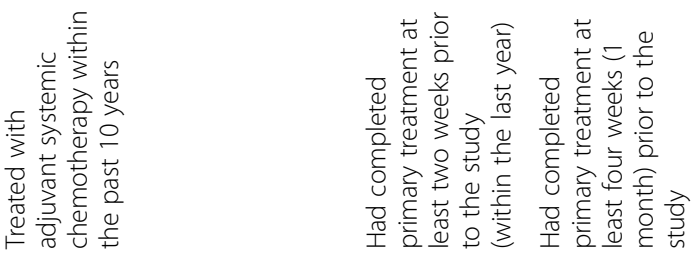

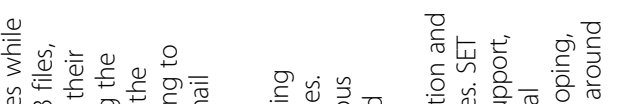

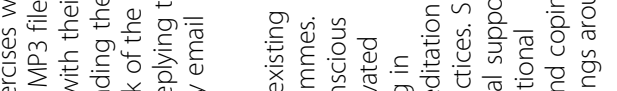

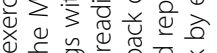

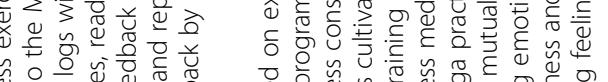

\section{这造造}

$+$

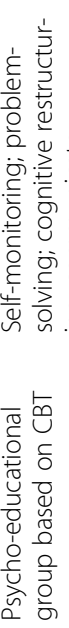

$\frac{ᄋ}{\frac{0}{0}}$

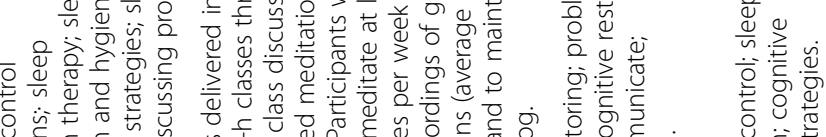
ठ

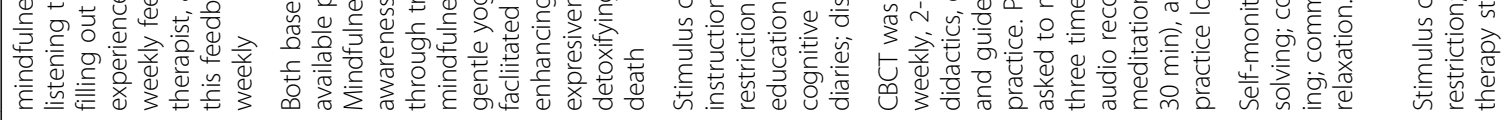
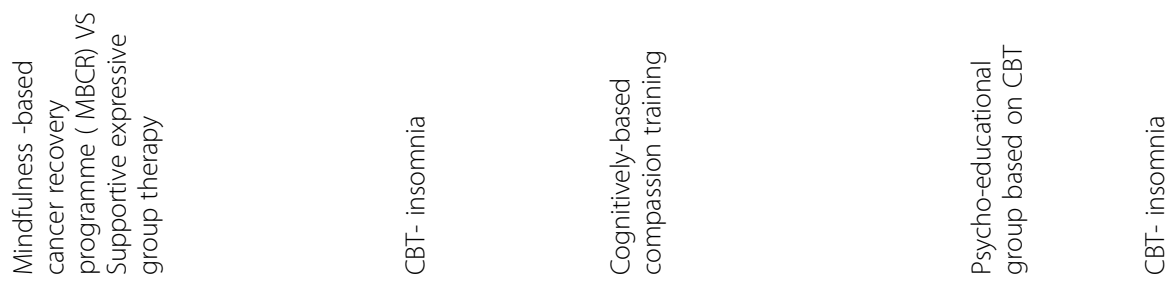

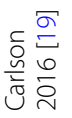

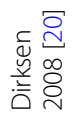

$\frac{n}{2}$
$\stackrel{n}{0}$
$\frac{n}{0}$
$\frac{0}{0}$

\begin{tabular}{|c|}
\hline 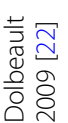 \\
\hline
\end{tabular}




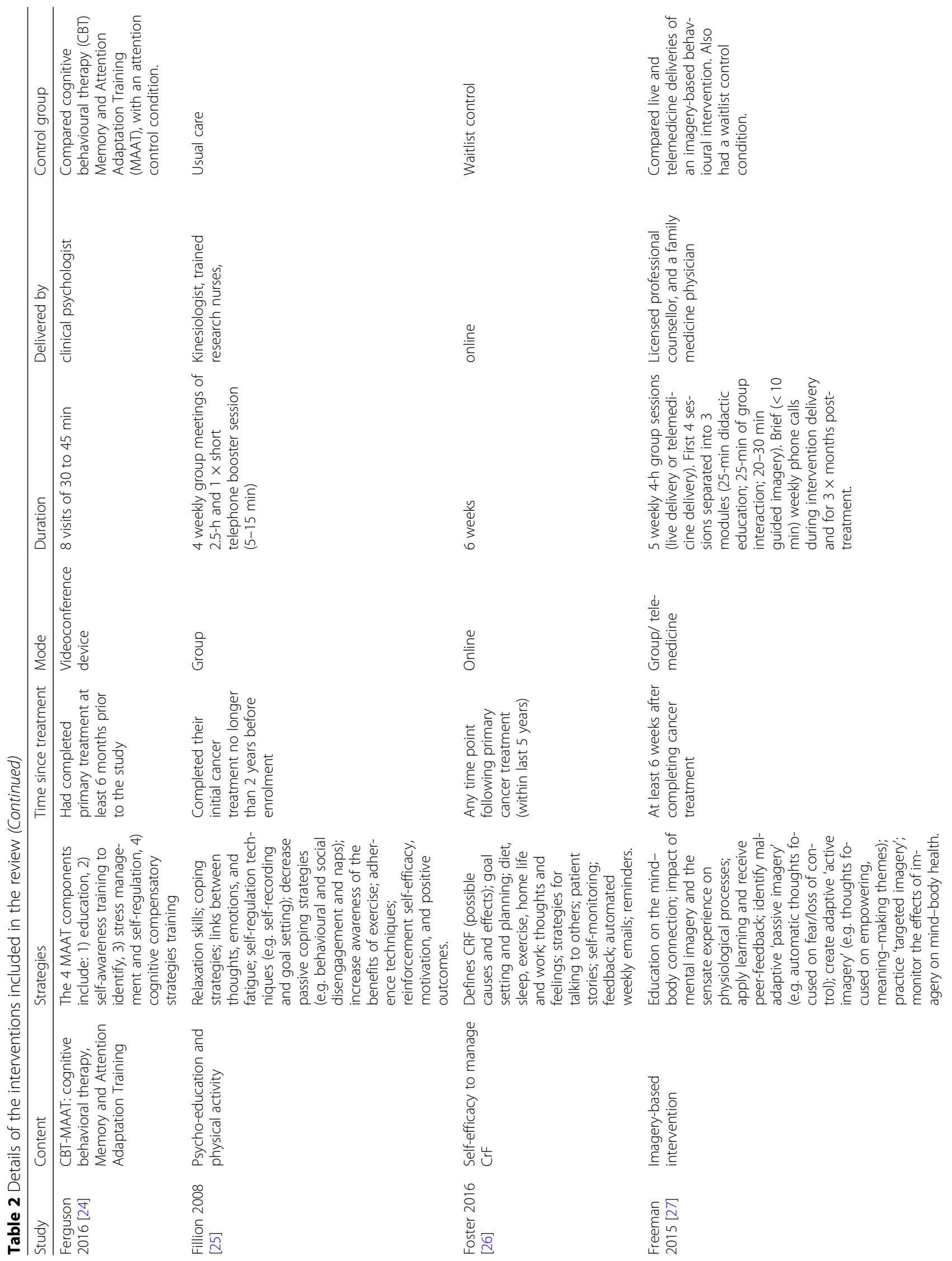




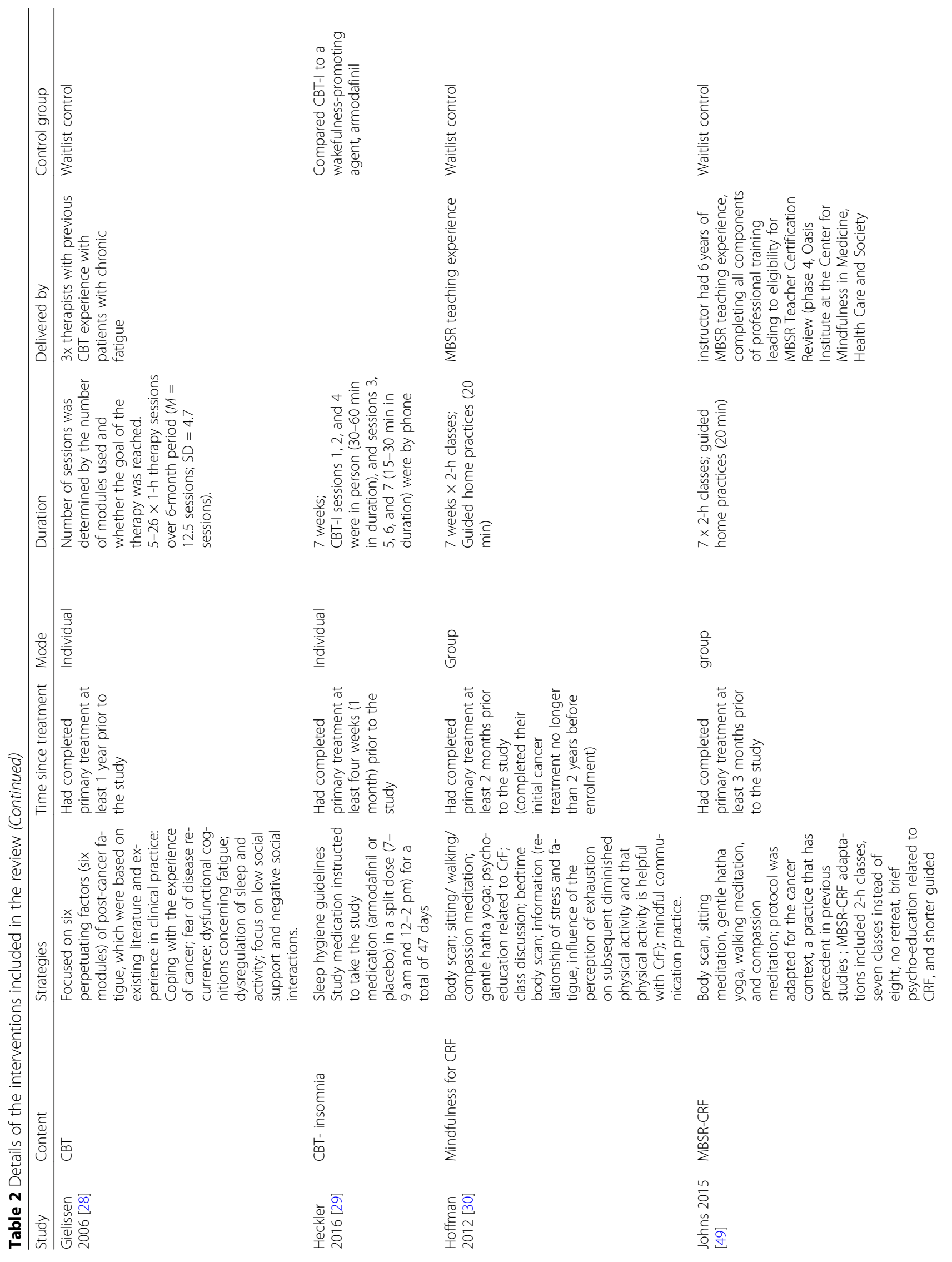




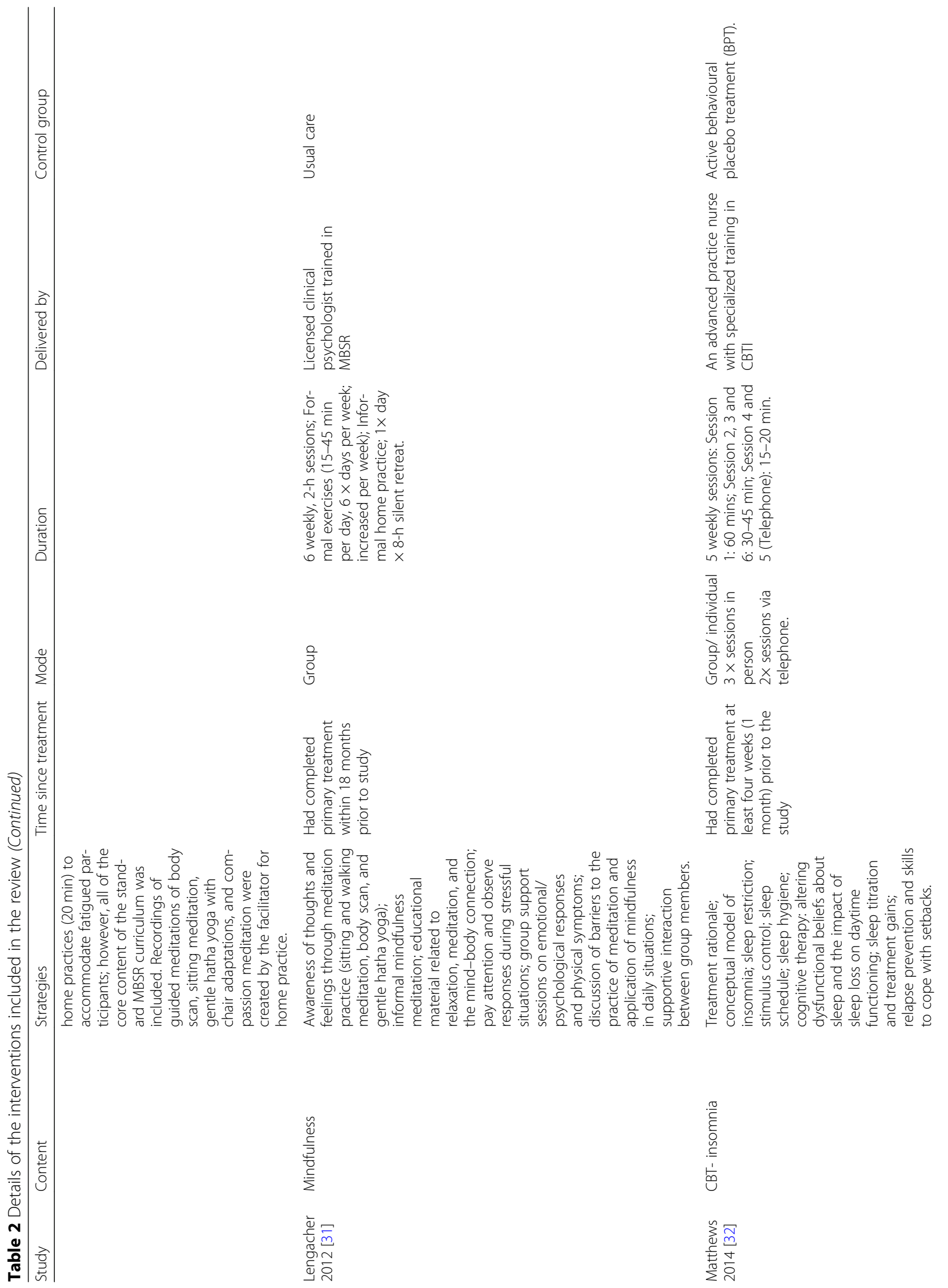



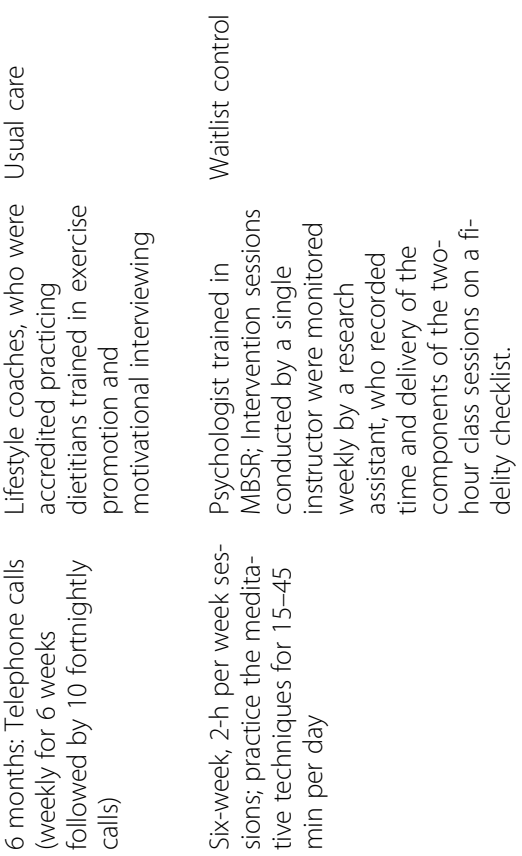

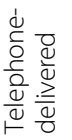

ํํำ
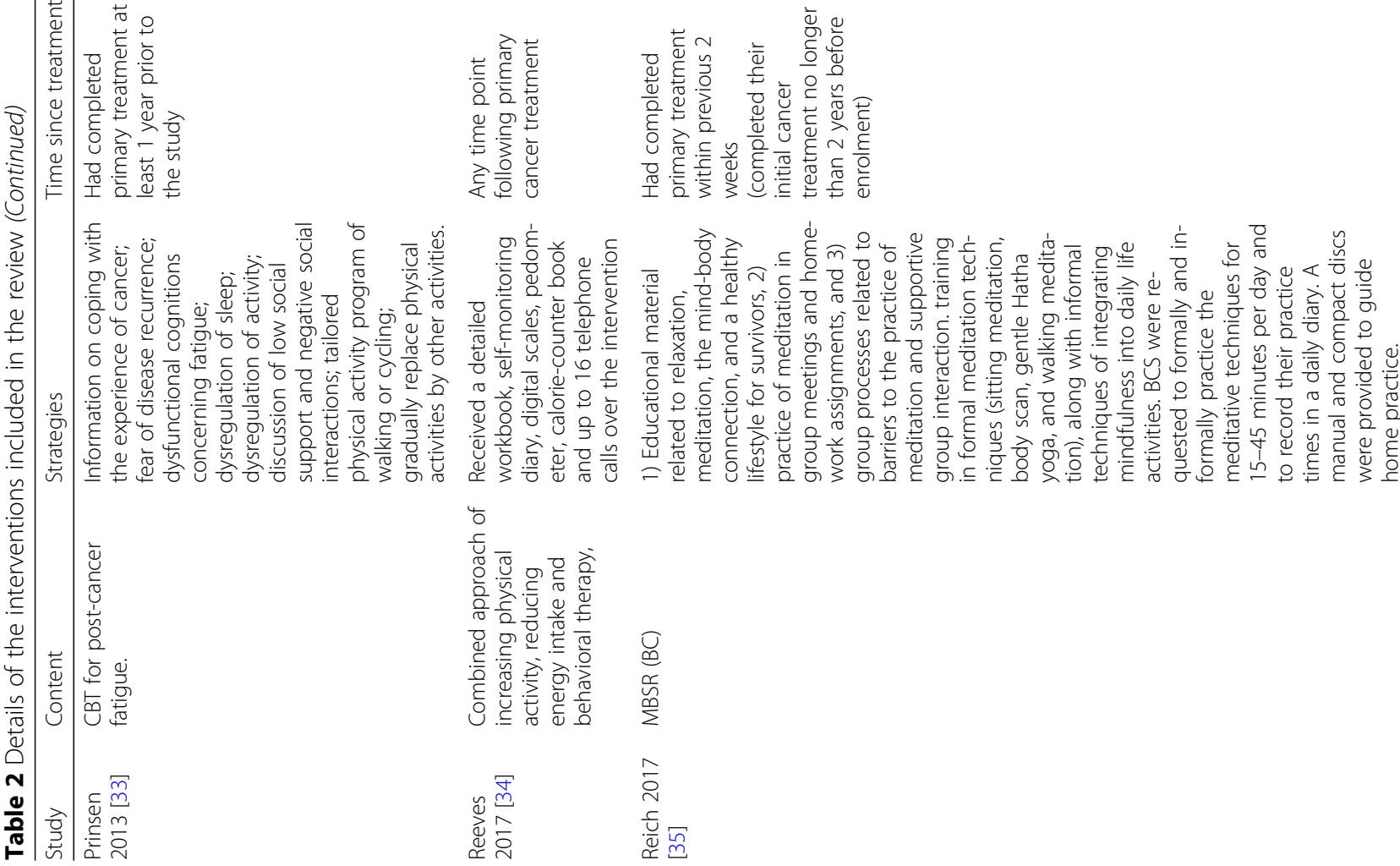


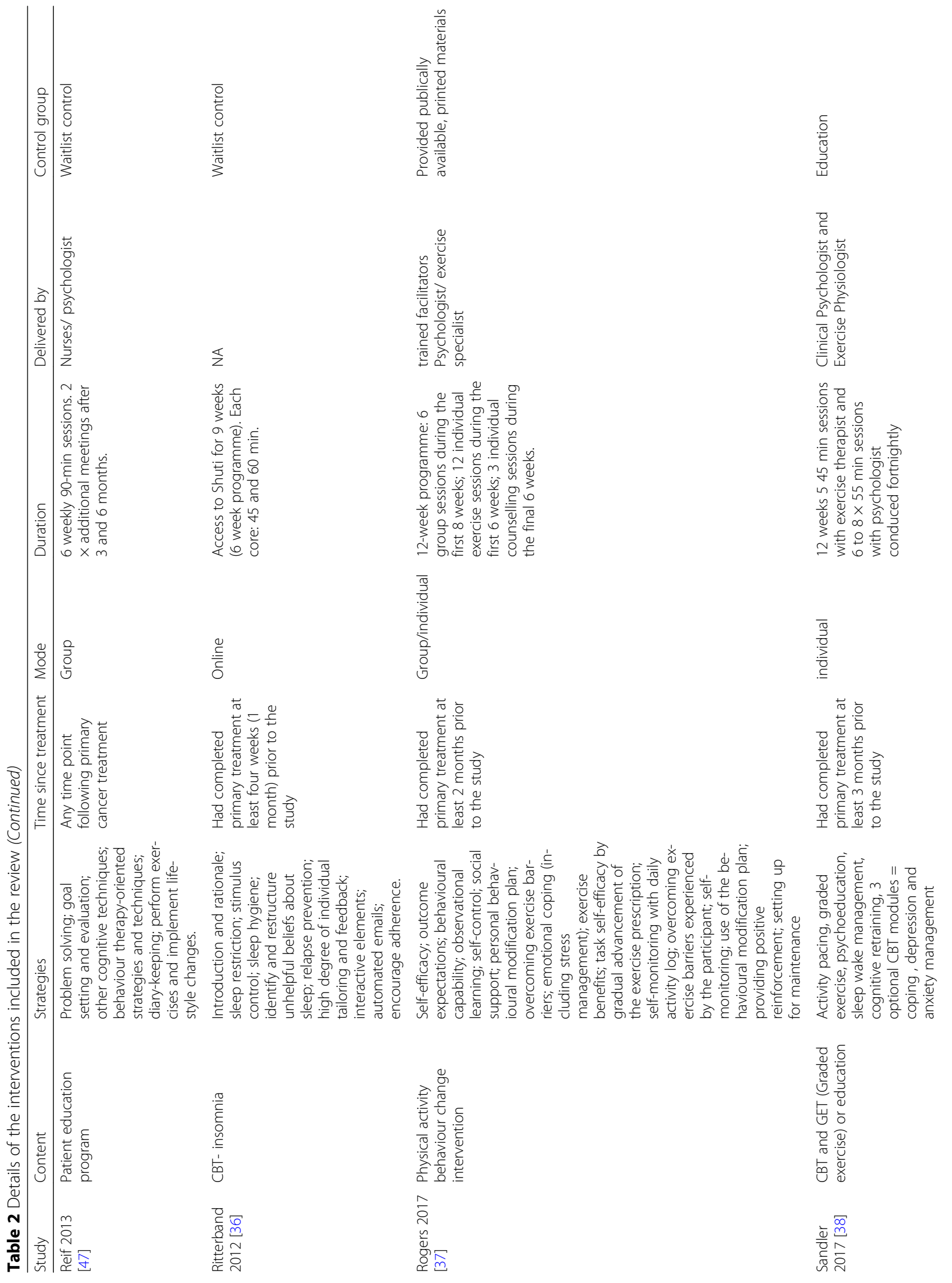




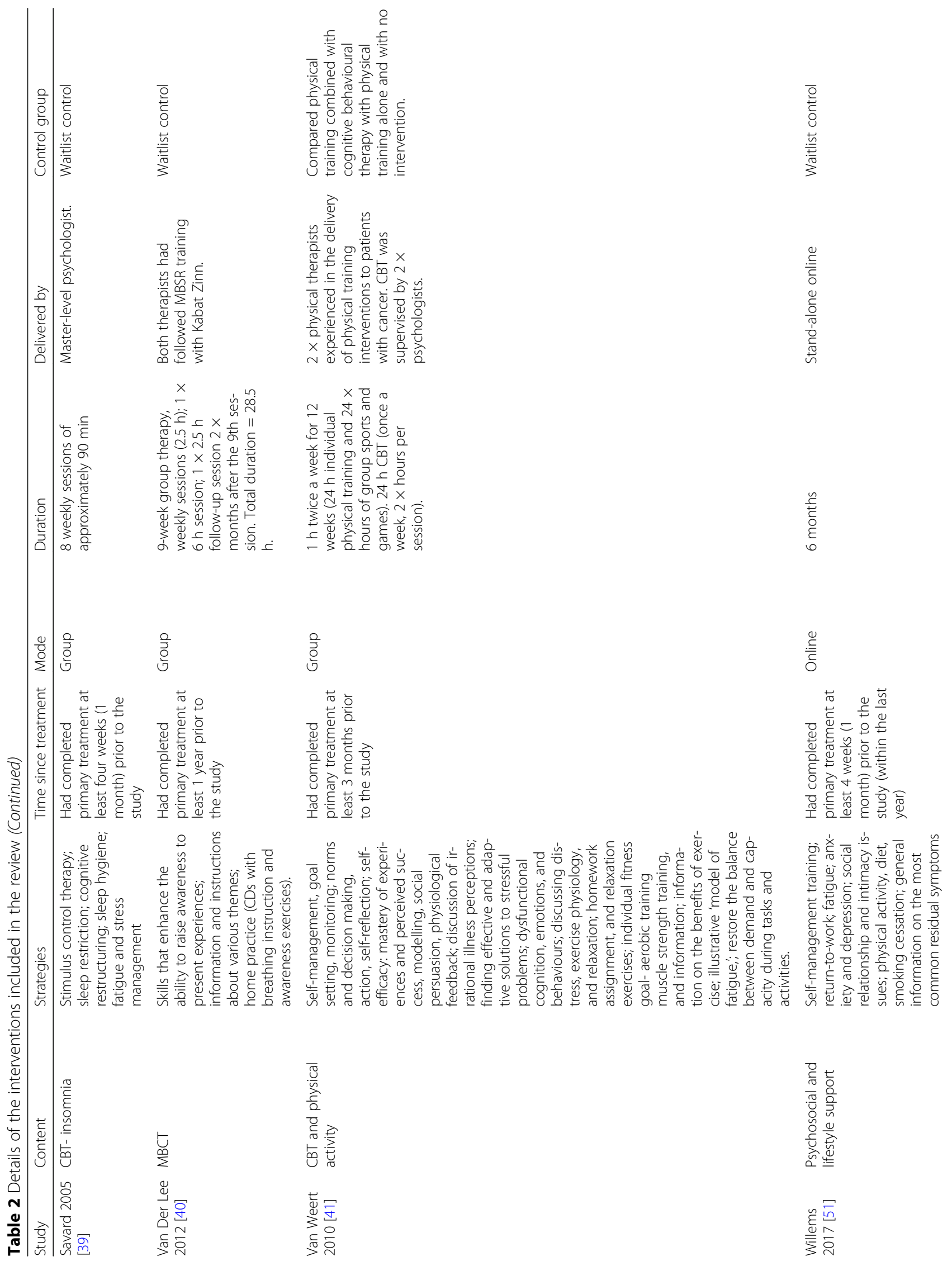




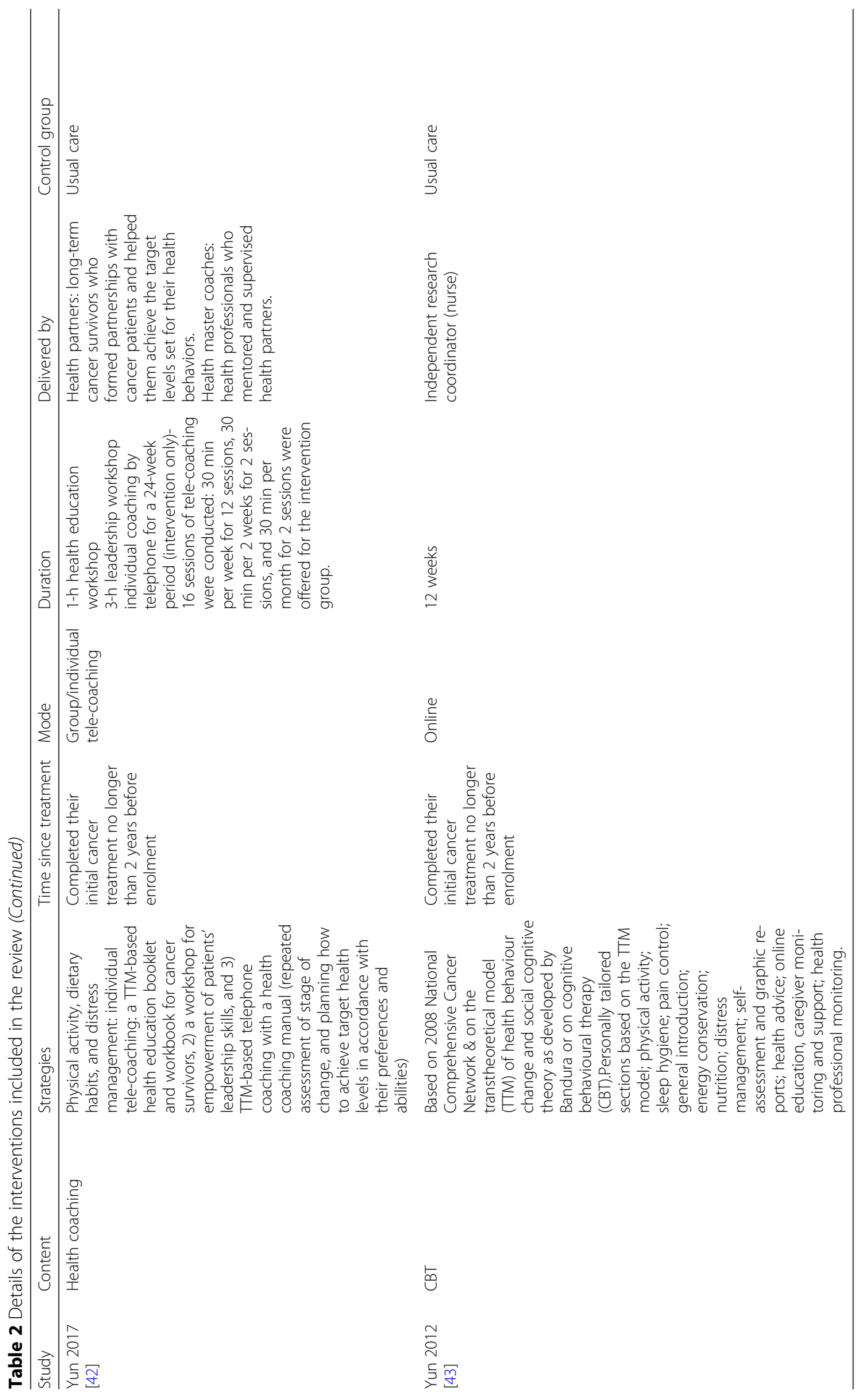




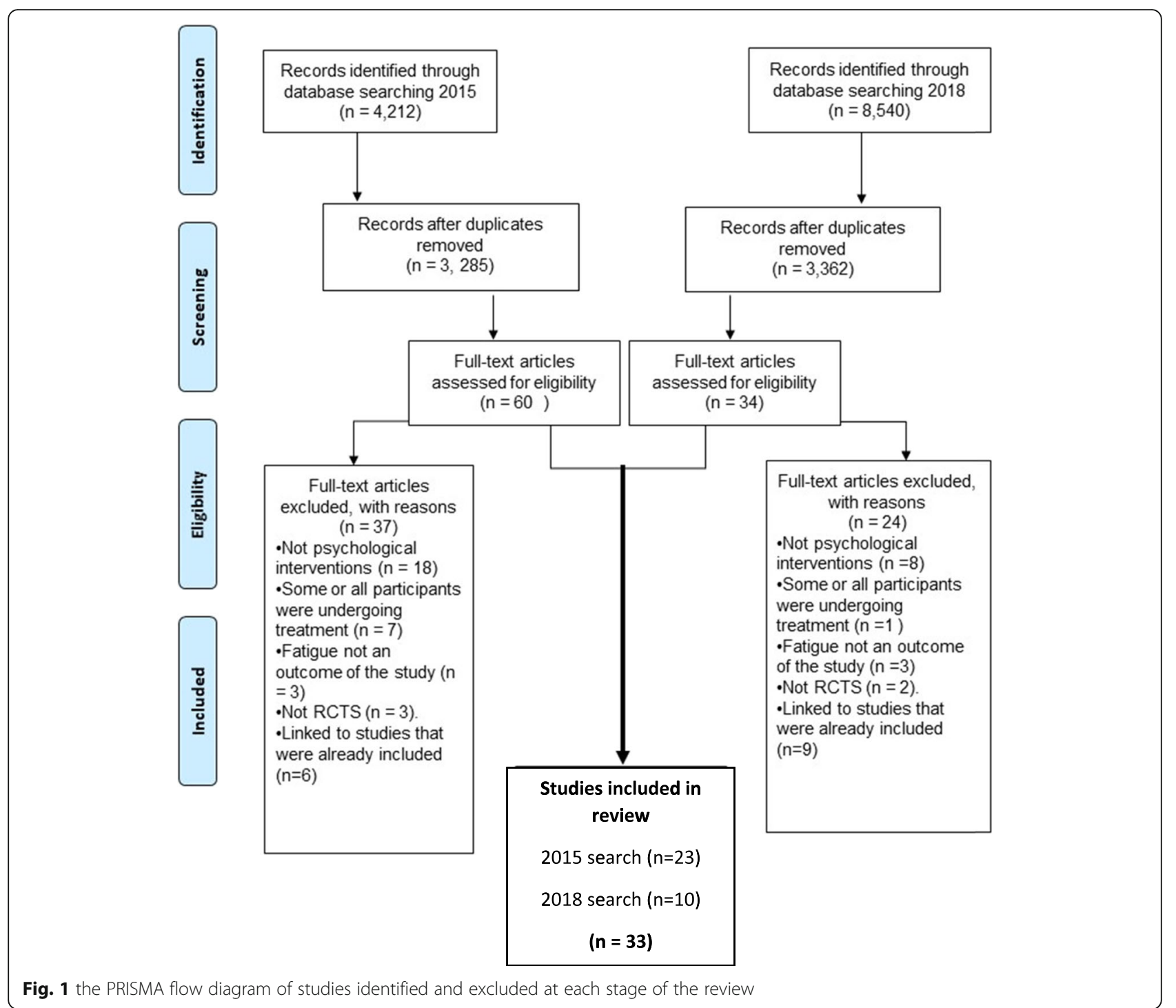

\section{Assessment of risk of bias in included studies}

The risk of bias of each trial was assessed as high risk, low risk or unclear risk as per recommendations provided in Chapter 8 of the Cochrane Hand book for Systematic Reviews of Interventions [44]. Further details regarding the risk of bias domains was provided in the study protocol [12].

\section{Quality of the evidence}

The Grading of Recommendations Assessment, Development, and Evaluation (GRADE) process was used to assess the evidence for the primary comparison of 'Psychological Interventions compared to usual care for Fatigue in cancer survivors'.

\section{Results}

Figure 1 depicts the PRISMA flow diagram of studies identified and excluded at each stage of the review. The initial literature search of seven databases in 2015 resulted in 4212 potentially relevant articles. Following exclusion of duplicates, 3,285 articles remained. The titles and abstracts of these articles were screened and 60 full-text articles were selected to be retrieved and reviewed in detail. Following review of the full-text papers, a further 37 studies were excluded and 23 RCTs fulfilled all eligibility criteria for inclusion.

The updated search in 2018 resulted in 8540 potentially relevant articles. Once duplicates and studies prior to 2014 were removed, 3362 studies published were assessed for inclusion. Thirty-four full-text articles were reviewed, eight of which had already been included or were follow-up studies associated with papers included in the original review. Ten new papers were added to the review.

In total, 33 RCTs fulfilled all eligibility criteria for inclusion. A full description of these studies can be seen in Tables 2 and 3. 
Table 3 Summary of findings for the main comparisons

\begin{tabular}{|c|c|c|c|c|c|c|}
\hline Study & Measure used to assess fatigue & Total & $\begin{array}{l}n \\
\text { intervention }\end{array}$ & $\begin{array}{l}n \\
\text { Control }\end{array}$ & $\begin{array}{l}\text { Final follow- } \\
\text { up }\end{array}$ & Finding \\
\hline $\begin{array}{l}\text { Bantum } 2014 \\
{[14]}\end{array}$ & Brief Fatigue Inventory (BFI) & 303 & 156 & 147 & 6 months & $\begin{array}{l}p=0.56 \text { Effect size }=0.17 \text { (Calculated by taking the } \\
\text { differences of the means at } 6 \text { months predicted from } \\
\text { the model, including adjustment factors, divided by th } \\
\text { standard deviation for the difference computed from } \\
\text { the within and between subject variance components } \\
\text { Control group, } \\
\text { - Baseline }(n=176) \text {; mean }(95 \% \mathrm{Cl})=40.8(38.9-42.8) \\
\text { - Month } 6(n=156) \text {; mean }(95 \% \mathrm{Cl})=40.7(38.7-42.8) \\
\text { Intervention group } \\
\text { - Baseline ( } n=176) \text {; mean }(95 \% \mathrm{Cl})=39.0(37.0-40.9) \\
\text { - Month } 6(n=147) \text {; mean }(95 \% \mathrm{Cl})=36.4(34.2-38.5)\end{array}$ \\
\hline
\end{tabular}

Bennett 2007 Schwartz Cancer Fatigue Scale $56 \quad 28$ [15]

Blaes 2016

[16]

Functional Assessment in

cancer Therapy-Fatigue ( FACT-

F)

Bower 2015 Fatigue Symptom Inventory

[17]

Bruggeman-

Everts 2017

[18]

Checklist Individual Strength -

Fatigue Severity [CIS-FS]

subscale
Carlson 2013 POMS

(2016) [19,

45]

Dirksen 2008 Profile of Mood States Fatigue/

[20] Inertia Subscale (POMSF/I)
Dodds 2015 Medical Outcomes Study Short

[21] Form 12-Item HealthSurvey (SF12)

POMSF/I and EORTC Fatigue
$167 \quad 81$

81

86

158

6 and 12 months

later.

2 weeks

$28 \quad 16$

12

4 weeks

95\% Cl [1.5; 9.6];

1-month FU 0.3

$95 \% \mathrm{Cl}[-4.2 ; 4.9]$ no significant differences at the 4week follow-up.

Comparison of change scores between randomisation arms (Group: $\mathrm{n}=81$; Control: $\mathrm{n}=87$ )

POMS fatique

- Group: E1 Mean (SD) 10.01 (7.38) ; E3 Mean (SD) 6.86

(5.58); Intra-subject $p=-0.069 \mathrm{Eta}^{2}=0.02$

- Control: E1 Mean (SD) 8.78 (6.85); E3 Mean (SD) 8.87

(6.84) Inter-subject $p=0.370 \mathrm{Eta}^{2}=0.01$ 
Table 3 Summary of findings for the main comparisons (Continued)

\begin{tabular}{|c|c|c|c|c|c|c|}
\hline Study & Measure used to assess fatigue & Total & $\begin{array}{l}n \\
\text { intervention }\end{array}$ & $\begin{array}{l}n \\
\text { Control }\end{array}$ & $\begin{array}{l}\text { Final follow- } \\
\text { up }\end{array}$ & Finding \\
\hline & & & & & & $\begin{array}{l}\text { - Time X group } p=0.000 \mathrm{Eta}^{2}=0.07 \\
\text { EORTC Fatigue } \\
\text { - Group: E1 Mean (SD) } 2.24(0.81) ; \mathrm{E} 3 \text { Mean (SD) } 2.08 \\
\text { (0.73) Intra-subject } p=0.834 \mathrm{Eta}^{2}=0.00 \\
\text { - Control E1 Mean (SD) } 2.09(0.68) ; \mathrm{E3} \text { Mean (SD) } 2.14 \\
\text { (0.77) } \\
\text { - Inter-subject } p=0.408 \mathrm{Eta}^{2}=0.00 \\
\text { - Time X group } p=0.036 \mathrm{Eta}^{2}=0.03 \\
\text { A greater reduction of negative affects and } \\
\text { improvement in positive affects and in quality of life } \\
\text { functional or symptom scales were observed in the TC } \\
\text { compared with the CG. This concerned the POMS } \\
\text { fatigue (7\% of the variance explained by the model } \\
\text { including the time/group interaction term) and the } \\
\text { EORTC QLQ-C30 fatigue (3\%). }\end{array}$ \\
\hline $\begin{array}{l}\text { Espie } 2008 \\
\text { [23] }\end{array}$ & FSI & 150 & 100 & 50 & 6 months & $\begin{array}{l}p<0.001 \text { (Standardized Effect }=-0.82 \text { ) } \\
\text { CBT participants had reduced symptoms of fatigue } \\
\text { relative to TAU. } \\
\text { FSI Interference } \\
\text { Post-Treatment } \\
\text { - Standardized Effect }-0.81 \\
\cdot 95 \% \mathrm{Cl}-1.20 \text { to- } 0.42 \\
\cdot p<0.001 \\
6 \text {-Month follow-up } \\
\cdot \text { Standardized Effect }-0.82 \\
\cdot 95 \% \mathrm{Cl}-1.22 \text { to }-0.42 \\
\cdot p<0.001\end{array}$ \\
\hline
\end{tabular}

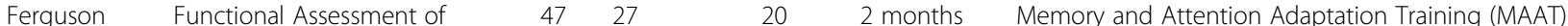
2016 [24] Chronic Illness Therapy-Fatigue [FACIT-F]

[25]

Multidimensional Fatigue

$87 \quad 44$

Inventory

Foster 2016

[26]

Freeman

2015 [27]
Brief Fatigue Inventory (BFI)

FACIT-Fatigue and Scale (FACIT$F$, version 4)
15983

118

71
Fatigue severity subscale of the $98 \quad 50$ CIS
Memory and Attention Adaptation Training (MAAT) and Supportive Therapy (ST) participants did not differ with regard to fatigue (FACIT-F) at the post-treatment
$(\mathrm{F}(1,28), 0.072 ; p=0.79)$ or 2 -month $((\mathrm{F}(1,28), 2.35 ; p=$ $(F(1,28), 0.072 ; p=0.79)$ or 2 -month ( $(F(1,28), 2.35 ; p$
$0.14)$. The Cohen's d effect sizes for, fatigue at the 2 month follow-up time point suggested that MAAT participants demonstrated sustained clinical gains compared with ST participants (0.46)

Marginal Group x Time interaction effects: $p=0.07$; Cohen $d=0.36$

Significant time main effects: $p=0.0001$; Cohen $d=$ 0.69

Significant group main effects: $p=0.03$; Cohen $d=$ 0.49

Results showed that participants in the intervention group

showed greater improvement in fatigue.
12 weeks

$47 \quad 3$ months
T1 Group effect $(95 \%$ Cl) $0.514(-0.084,1.112) p=0.09$ T2 Group effect $(95 \%$ Cl) $0.106(-0.427,0.638) p=0.70$

Group effect $p$ value $=0.002$

Time effect $p$ value $=0.084$

Group $\times$ time effect $p$ value $=0.321$

The Bonferroni method was used to correct for multiple comparisons, and alpha was adjusted to 0.01 . Linear multilevel modelling analyses revealed less fatigue, cognitive dysfunction, and sleep disturbance for Live Delivery and Telephone Delivery compared with WL across the follow-up ( $p$ 's $<0.01$ ). Changes in fatigue, cognitive dysfunction, sleep disturbance, and health-related and breast cancer-related QOL were clinically significant. There were no differences between LD and TD.

$48 \quad 6$ months
Patients in the intervention condition reported a significantly greater decrease than patients in the waiting list condition in fatigue severity (difference, $13.3 ; 95 \% \mathrm{Cl}, 8.6$ to 18.1$)$ 
Table 3 Summary of findings for the main comparisons (Continued)

\begin{tabular}{|c|c|c|c|c|c|c|}
\hline Study & Measure used to assess fatigue & Total & $\begin{array}{l}n \\
\text { intervention }\end{array}$ & $\begin{array}{l}n \\
\text { Control }\end{array}$ & $\begin{array}{l}\text { Final follow- } \\
\text { up }\end{array}$ & Finding \\
\hline $\begin{array}{l}\text { Heckler } 2016 \\
\text { [29] }\end{array}$ & $\begin{array}{l}\text { Brief Fatigue Inventory (BFI)/ } \\
\text { FACIT-F }\end{array}$ & 96 & 47 & 49 & $\begin{array}{l}7 \text { weeks } \\
\text { (post } \\
\text { intervention) }\end{array}$ & $\begin{array}{l}\text { CBT and placebo } p=0.0005(95 \% \mathrm{Cl})[-2.22,-0.74] \\
\text { CBT and placebo } p \leq 0.0001 \text { ( } 95 \% \text { CI) }[5.57,12.90] \\
\text { CBT-I effect }(95 \% \text { CI) for BFI was }-1.00(-1.64,-0.37) \text {, } \\
p=0.0024 \text {, meaning that CBT-I led to a mean change } \\
\text { one unit less than no CBT-I. } \\
\text { The CBT-I effect ( } 95 \% \text { Cl) for FACIT-Fatigue was } 7.16 \\
(3.68,10.64), p<0.0001 \text {, meaning that CBT-I led to a } \\
\text { mean change seven units higher than no CBT-I. } \\
\text { No statistically significant change between post- } \\
\text { intervention and follow-up; } p=0.294 \text { (BFI), } p=0.145 \\
\text { (FACIT-Fatigue). }\end{array}$ \\
\hline
\end{tabular}

$\begin{array}{llllll}\text { Hoffman } & \text { pOMSF/l } & 214 & 103 & 111 & \begin{array}{l}12-14 \\ \text { 2012 [30] }\end{array}\end{array}$

Johns 2015 Fatigue Symptom Inventory

[49]

Lengacher

Symptom Inventory (MDASI)

2012 [31]

Matthews

Piper Fatigue Scale

Prinsen 2013

[33]

Checklist Individual Strength

(CIS-fatigue)

$35 \quad 18$

17

$37 \quad 23$

14
1 month

6 months

Reeves 2017 FACIT

[34]
$90 \quad 45$

45
There were statistically significant differences between treatment groups for POMS fatigue $p=0.002$ [8 weeks only]

Difference Between Groups at T2 adjusted for baseline mean $=-2.68 ; 95 \% \mathrm{Cl}=[-4.31$ to -1.04$]$

Difference between groups at T3 adjusted for baseline mean $=-1.8495 \% \mathrm{Cl}=[-3.45$ to -0.22$]$

Interaction time $\mathrm{X}$ treatment group, $P .324$

Significantly greater improvements in fatigue interference than wait-list controls. The magnitude of the effect of MBSR on this and other fatigue outcomes including fatigue severity and vitality was large at the end of the intervention and 1 month later. improvements in all symptoms were maintained for at least 6 months beyond the completion of the MBSR course for both groups after their respective courses. T2

FSI interference

$p^{*} \leq 0.001$ Pooled SD $=1.73$ Effect size $=-1.4395 \% \mathrm{Cl}$

effect size $=[1.96,-0.90]$

FSI severity

$p^{*} \leq 0.001$ Pooled SD $=1.64$ Effect size $=-1.5595 \% \mathrm{Cl}$ effect size $=[-2.09,-1.01]$

T3

FSI interference

$p^{*} \leq 0.001$ Pooled SD $=2.01$ Effect size $=-1.3495 \% \mathrm{Cl}$

effect size $=[1.88,-0.81]$

FSI severity

$p^{*} \leq 0.001$ Pooled SD $=1.51$ Effect size $=-1.5495 \% \mathrm{Cl}$ effect size $=[-2.10,-0.97]$

$p<0.5$

$\mathrm{P}$ (between-group post-assessment) $p=0.05$

At post-intervention, the MBSR(BC) group showed greater improvement across symptoms, and especially symptom interference items, compared to the control group. For the MBSR(BC) group, statistically-significant reductions $(p<0.01)$ were observed for fatigue.

$p=0.76 d=0.2$

No group differences in improvement were noted relative to fatigue.

CBT resulted in a significantly larger decrease in fatigue severity compared to a period of waiting for therapy. After 6 months of follow-up, patients who underwent CBT, with a mean of $12.0 \pm 5.0$ individual sessions, showed a significantly larger change in fatigue scores than patients in the waiting list group $(p<0.001$, respectively $-49.0 \pm 23.0 \%$ and $-16.4 \pm 25.0 \%)$. Baseline to follow-up (within group) $p<0.001 p=$ 0.022

Only the intervention arm showed significantly improved

Fatigue- Mean change $(95 \% \mathrm{Cl})=3.0(0.7,5.3) p<0.01$ Intervention - usual care- No statistically significant 
Table 3 Summary of findings for the main comparisons (Continued)

\begin{tabular}{lllll}
\hline Study Measure used to assess fatigue & Total & $n$ & $n$ & Fina \\
& intervention & Control up \\
\hline
\end{tabular}

\section{Reich 2017/}

Lengacher

$2016[35,46]$
Fatigue Symptom Inventory
$303 \quad 155$

148
12 weeks
Reif 2013 [47] Fatigue Assessment

Questionnaire (FAQ) and

Fatigue subscale of the EORTC-

QLQ-C30

$234 \quad 120$

114

6 months

Ritterband 2012 [36]
Multidimensional Fatigue Symptom Inventory- Short Form (MFSI-SF)
Rogers 2017 Fatigue Symptom Inventory

[37]
$222 \quad 110$

112

3 months
Final follow- Finding

intervention effects were observed

Mean difference $(95 \% \mathrm{Cl})=1.1(-2.4,4.5)$

$p=0.527$

$\mathrm{MBSR}(\mathrm{BC})$ demonstrated greater symptom improvement in fatigue (severity and interference; $p<$ 0.01).

Effect sizes (Cohen's d) were between 0.27 and 0.23. A majority of improvements in fatigue occurred during the MBSR(BC) training, with little change occurring during the follow-up period (6 to 12 weeks). Fatigueseverity (FSI) $p=0.002 \mathrm{~T} 2$ week $d=0.3395 \% \mathrm{Cl}[0.13$ to $0.54]$ T3 week $d=0.2795 \% \mathrm{Cl} 120.07$ to $0.47 \mathrm{Fa}$ tigue-interference (FSI) $p=0.006 \mathrm{~T} 2$ week $d=0.3$ $95 \% \mathrm{Cl}[0.10$ to 0.51 ] T3 week $d=0.2395 \% \mathrm{Cl}[0.02$ to 0.43]

FAQ : Significant reduction in intervention group: $(F=$ $76.510, p<0.001, \eta 2=0.248$ ). The control group showed almost no change in CRF levels over time. In the repeated measures ANOVA, this difference was statistically significant for the group by time interaction $(\mathrm{F}=76.51, p<0.001)$. The partial $\eta 20 f 0.248$ indicates a large effect.

QLQ-C30 fatigue subscale: the IG showed a reduction from 75.37 (19.39) to 40.74 (30.60) while the values in the CG remained about the same $(F=57.837$, partial $\eta 2=0.2, p<0.001)$. This finding confirms the results of the FAQ.

$p<0.01$

Overall adjusted ES $(d)=1.16$

A significant group $x$ time interaction was found for the overall measure of fatigue, MFSI-SF $\left(F_{1,26}=13.88, p\right.$ $<0.01)$. Participants in the Internet group had significantly improved fatigue scores from 22.86 to 9.50 ( $t(13)$ $=3.63, p<0.01)$; control participants' scores did not improve over time, changing from 13.71 to $19.79(t(13)=$ - 1.64, $p=0.12$ ). Several MFSI-SF subscales also had significant group $\times$ time interactions, including general fatigue $\left(F_{1,26}=9.46, p<0.01\right)$, mental fatigue $\left(F_{1,26}=\right.$ $.65, p<0.01)$, and vigor $\left(F_{1,26}=14.79, p<0.01\right)$, with Internet participants showing improvements compared with control participants in all cases. Although some subscales lacked significant group $\times$ time interactions (physical fatique, $p=0.11$; emotional fatique, $p=0.08$ ), adjusted ES for the fatigue variables ranged from a low of 0.47 to a high of 1.63 , indicating a SHUTi treatment effect for fatigue.

BEAT Cancer significantly reduced fatigue intensity at both time points (mean between group difference [M] $=-0.61 ; 95 \% \mathrm{Cl}=-1.04$ to -0.19 ; effect size $[d]=-$ $0.32 ; p=.004$ at $\mathrm{M} 3$ and $\mathrm{M}=-0.46 ; 95 \% \mathrm{Cl}-0.89$ to $-0.03 ; d=-0.26 ; p=.038$ at M6).

Significant and greater reductions in fatigue interference

occurred $(\mathrm{M}=-0.84 ; 95 \% \mathrm{Cl}=-1.26$ to $-0.43 ; d=-$ 0.40;

$p<.001$ at $\mathrm{M} 3$ and $-0.66 ; \mathrm{Cl}-1.08$ to $-0.24 ; d=-$ $0.35 ; p=.002$ at M6).

Fatigue severity improved in all subjects from a mean of $5.2(-3.1)$ at baseline to $3.9(-2.8)$ at 12 weeks, suggesting a natural history of improvement. Clinically significant improvement was observed in 7 of 22 subjects in the intervention group compared with 2 of 24 in the education group $(p<0.05)$

The whole cohort reported improvements in fatigue 
Table 3 Summary of findings for the main comparisons (Continued)

\begin{tabular}{|c|c|c|c|c|c|c|}
\hline Study & Measure used to assess fatigue & Total & $\begin{array}{l}n \\
\text { intervention }\end{array}$ & $\begin{array}{l}n \\
\text { Control }\end{array}$ & $\begin{array}{l}\text { Final follow- } \\
\text { up }\end{array}$ & Finding \\
\hline & & & & & & $\begin{array}{l}\text { scores between baseline and } 12 \text { weeks (Mdiff }=-1.27 \text {; } \\
95 \% \mathrm{Cl}-2.52 \text { to }-0.03 ; p<0.05 \text { ) and } 24 \text { weeks (Mdiff } \\
=-1.51 ; 95 \% \mathrm{Cl}-2.84 \text { to }-0.18 ; p<0.05 \text { ). } \\
\text { Change scores differed significantly in favour of the } \\
\text { intervention }(\mathrm{M}=2.55, \mathrm{SD}=3.77 ; t(36)=-2.56 ; p< \\
0.05) \text { at } 12 \text { weeks in comparison to the education arm } \\
(\mathrm{M}=0.10 ; \mathrm{SD}=2.55) \text { but not at follow up (Mdiff }= \\
1.56 ; 95 \% \mathrm{Cl}-3.77 \text { to } 0.48 ; p=0.13 \text { ). } \\
\text { These groupwise changes indicate an effect size in the } \\
\text { CBT/GET group of } d=0.79 \text {, compared with } d=0.04 \text { in } \\
\text { the education arm. }\end{array}$ \\
\hline
\end{tabular}

Savard 2005 Multidimensional Fatigue

[39] Inventory (MFI)

Van Der Lee 2012 [40] Inventory (MFI)- General fatigue
Multidimensional Fatigue

$57 \quad 27$

30

12 months pre- and post-treatment on fatigue $(F 1,158=11.70 ; p<$ .001), No significant difference was detected between post-treatment and the follow-up evaluations. Therapeutic effects were well maintained up to 12 months after the intervention and generally were clinically significant.

Pooled data

$(n=57)$

3-month follow-up : adjusted mean $=2.33 ; 95 \% \mathrm{Cl}=$

2.15 to 2.51

6-month follow-up: adjusted mean $=2.25 ; 95 \% \mathrm{Cl}=$

2.07 to 2.43

12-month follow-up: adjusted mean $=2.18 ; 95 \% \mathrm{Cl}=$ 1.98 to 2.38

$p<0.001$

At post-treatment measurement the proportion of clinically improved participants was 30\%, versus $4 \%$ in the waiting list condition $\left(X^{2}(1)=56.71 ; p=0.007\right)$.

The mean fatigue severity score at post-measurement was significantly lower in the intervention group $(95 \% \mathrm{Cl}=33.2-37.9)$ than in the waiting list group (95\% $\mathrm{Cl}=40.0-47.4)$ controlled for pre-treatment level of fatigue. The effect size for fatigue is $0.74(d=$ (mean post intervention-mean post control)/pooled SD).

The treatment effect was maintained at 6-month follow-up. At follow up 39\% of the participants in the intervention group

showed clinically relevant improvement in fatigue severity.

Van Weert

Multidimensional Fatigue

Inventory (MFI)- General fatigue

20976

2010 [41] [48]
409188 CIS
2216 months

12 months
In comparison with the WLC group, the PT group showed more reduction in 4 domains of fatigue, whereas the PT+CBT group showed more reduction in one domain only. Finally, the results showed that physical training combined with CBT and physical training alone were equally effective in reducing fatigue. Thus, CBT did not seem to contribute additional positive effects on fatigue to the benefits of physical training.

$\mathrm{PT}+\mathrm{CBT}$ (WLC = Reference) between-group change General fatigue $(95 \% \mathrm{Cl})=-1.3(-3.1$ to 0.4$)$ Physical fatigue $(95 \% \mathrm{Cl})=-2.7(-4.5$ to -1.0$) p<$ 0.01 .

Mental fatigue $(95 \% \mathrm{Cl})=-0.5(-2.3$ to 1.2$)$

Reduced motivation $(95 \% \mathrm{Cl})=-0.6(-2.1$ to 1.0$)$

Reduced activation $(95 \% \mathrm{Cl})=-0.9(-2.6$ to 0.8$)$

The intervention was effective in reducing fatigue ( $B$

$=-4.36, p=0.020, d=0.21$ ).

Adjusted: 6 months

$p=0.030$

$95 \% \mathrm{Cl}[-7.87$ to -0.39$](d=0.21)$

Adjusted: 12 months

$p=1.000$

$95 \% \mathrm{Cl}[-3.88$ to 3.88$](d=0.04)$ 
Table 3 Summary of findings for the main comparisons (Continued)

\begin{tabular}{|c|c|c|c|c|c|c|}
\hline Study & Measure used to assess fatigue & Total & $\begin{array}{l}n \\
\text { intervention }\end{array}$ & $\begin{array}{l}n \\
\text { Control }\end{array}$ & $\begin{array}{l}\text { Final follow- } \\
\text { up }\end{array}$ & Finding \\
\hline & & & & & & $\begin{array}{l}\text { Between- group differences at } 12 \text { months from } \\
\text { baseline on emotional ( } p=.611, d=0.04 \text { ) were non- } \\
\text { significant } \\
\text { The intervention group remained fairly stable in fatigue } \\
\text { between } 6 \text { and } 12 \text { months from baseline, but the } \\
\text { control group slightly improved over time, leading to } \\
\text { non-significant group differences at } 12 \text { months from } \\
\text { baseline. }\end{array}$ \\
\hline $\begin{array}{l}\text { Yun } 2017 \\
{[42]}\end{array}$ & EORTC QLQ-C30 fatigue score & 174 & 57 & 117 & 12 months & $\begin{array}{l}\text { From baseline to } 12 \text { months, the LP group, relative to } \\
\text { the UC group, showed a significantly greater decrease } \\
\text { in the EORTC QLQ-C30 fatigue score }(p=0.065) \\
3 \text { months: } p=0.21412 \text { months: } p \text { value }=0.010^{* *}\end{array}$ \\
\hline $\begin{array}{l}\text { Yun } 2012 \\
{[43]}\end{array}$ & $\begin{array}{l}\text { Brief Fatigue Inventory (BFI) and } \\
\text { Fatigue Severity Scale (FSS) }\end{array}$ & 273 & 136 & 137 & 3 months & $\begin{array}{l}\text { BFl: } \\
p<0.01 \\
95 \% \mathrm{Cl}-1.04 \text { to- } 0.27 \\
\text { Cohen's } d=0.29 \\
\text { FSS: } \\
p<0.01 \\
95 \% \mathrm{Cl}-0.78 \text { to }-0.21 \\
\text { Cohen's } d=0.27 \\
\text { Compared with the control group, the intervention } \\
\text { group had an improvement in fatigue as shown by a } \\
\text { significantly greater decrease in BFI global score }(-0.66 \\
\text { points; } 95 \% \mathrm{Cl}-1.04 \text { to }-0.27) \text { and FSS total score }(- \\
0.49 ; 95 \% \mathrm{Cl}-0.78 \text { to }-0.21) \text {. }\end{array}$ \\
\hline
\end{tabular}

In cases where more than one paper was published relating to the same study, the papers were assigned to one study. Five articles were found in the grey literature and full-texts were not available online. Study authors of each of these papers were contacted. Three study authors provided full-texts in preparation for publication. The other two papers were excluded at this point, as full-texts were not available. No articles were found in snowball search.

\section{Description of included studies}

Data were extracted from the included papers (see Table 2. for a description of the included studies). The 33 RCTs reported data on 4486 cancer survivors (2196 intervention and 2290 controls). The majority of studies were conducted in the USA [14-17, 20, 21, 24, 27, 29, $31,32,35-37,49,50]$. Six were carried out in the Netherlands $[18,28,33,40,41,48]$, three in the UK [23, 26, 30]. The remainder were conducted in Australia, [34, 38] Canada [39, 45], Germany [47], France [22, 25] and Korea [42, 43].

\section{Participants}

As per the inclusion criteria for this review, studies were required to include only those who have completed active medical treatment prior to taking part in the research. However, there was little consistency across the studies regarding the timing of the intervention in relation to time elapsed since completion of cancer treatment.

\section{Interventions}

Details of interventions can be seen in Table 2, including content, strategies employed, mode of delivery, duration, who delivered the intervention and the comparison or control group used. Twelve studies reported on the effects of a CBT intervention [20, 23, 24, 28, 29, 32, 33, $36,38,39,41,43]$, of which six were focused specifically on CBT for insomnia (CBT-i) [20, 23, 29, 32, 36, 39]. Over half of these $(n=5)$ were studies on CBT-I [20, 23, 29, 36, 39]. Two of the CBT interventions were combined with physical activity [38, 41]. Other studies incorporated CBT strategies into the intervention. Dolbeault et al. [22] reported on a psycho-educational intervention based on CBT and another study reported on a trial of Cognitively-Based Compassion Training [21]. Van der Lee et al. used a combination of CBT and mindfulness strategies in a trial on mindfulness-based cognitive therapy [40].

Seven studies [16, 17, 19, 30, 31, 35, 49] reported on mindfulness-based interventions. Two of the studies were specifically aimed at $\mathrm{CrF}[30,49]$, and three were focused on cancer $[16,19,35]$.

Bruggeman-Everts [18] compared ambulant activity feedback (AAF) and psychologist-guided web-based mindfulness-based cognitive therapy groups to a psychoeducational group, showing that the psychoeducation group was least effective at reducing fatigue. Other interventions included a patient education program [47], a physical activity behaviour change intervention [37] and a combined psycho-education and physical 
activity intervention [25]. Health coaching and motivational interviewing was employed in two studies [15, 42]. Freeman et al. tested an imagery-based intervention [27]. Three studies reported on lifestyle interventions $[14,34,51]$ and one online intervention aimed to enhance self-efficacy to manage problems associated with cancer-related fatigue following primary cancer treatment [26].

\section{Control group}

There was substantial heterogeneity in the comparison groups used within the trials. See Table 2 for further details

\section{Outcomes}

\section{Primary outcomes}

A variety of different measures were used to assess fatigue. The Brief Fatigue Inventory (BFI) was used in five studies $[14,17,26,29,43]$ and the Functional Assessment in Cancer Therapy-Fatigue (FACIT-F) was used in five studies [16, 24, 27, 29, 34]. Five studies used the Fatigue Symptom Inventory (FSI) [17, 23, 35, 37, 49] and the Multidimensional Fatigue Inventory (MFI) was used in four studies [25, 39-41]. Ritterband [36] used the short form of the Multidimensional Fatigue Symptom Inventory-Short Form(MFSI-SF). The Schwartz Cancer Fatigue Scale was used in one study [15]. Four studies $[18,28,33,48]$ employed the Checklist Individual Strength (CIS). The remaining studies used fatigue subscales of broader multi-dimensional measures. Three studies assessed fatigue using two different questionnaires. Yun et al. [43] used both the BFI and the Fatigue Severity Scale (FSS), whereas another study used the BFI in conjunction with the FACIT-F [29]. The third study used both the Fatigue Assessment Questionnaire (FAQ) and fatigue subscale of the EORTC-QLQ-C30 [47].

\section{Secondary outcomes}

Secondary outcomes of interest to this review were specified a priori in the study protocol [12] and are summarised in Additional file 1. These included mood (self-reported scales of depression, and/or anxiety or distress); global quality of life and functional impact of fatigue (self-report questionnaire measures assessing the impact of fatigue on quality of life and daily functioning); and fatigue self-efficacy. Most of the studies included a measure of mood, either as an outcome or a control variable. However, the mood outcomes were assessed by a wide range of psychometric tools which assessed various dimensions of mood including stress, depression, anxiety and distress. Many of the studies also included a measure of global quality of life (QoL) and functional impact of fatigue. Only two of the studies assessed self-efficacy in relation to coping with fatigue $[25,26]$.

In the review process, other frequently reported secondary outcomes that were not outlined in the review protocol were identified as relevant to this review. These outcomes of interest were insomnia or sleep quality and pain. Studies that assessed sleep quality or insomnia tended to be designed with the aim of impacting insomnia or quality of life after cancer treatment.

As with the measures used to assess fatigue, a variety of measures were used to assess mood-related variables, with some studies including more than one measure of mood. The most commonly used measures were the Hospital Anxiety and Depression Scale (HADS) [52], The Patient Health Questionnaire (PHQ) [53] (a measure of depression severity) and The Profile of Mood States (POMS) [54] (a measure of psychological distress). The State-Trait Anxiety Inventory (STAI) [55] was also used.

The two most commonly used scales to assess quality of life were the European Organisation for the Research and Treatment of Cancer Quality of Life Questionnaire Core 30 (EORTC QLQ-C30) [56] and the Functional Assessment of Cancer Therapy-General (FACT-G) [57]. In the study protocol, the reviewers aimed to delineate the concepts of 'global quality of life' and 'functional impact of fatigue' [12]. However, in line with Luckett et al. [58], this was not deemed appropriate in the final review. Both types of measures assess physical, emotional, social, and functional/role scales. The QLQ-C30 provides brief scales for cognitive functioning, financial impact and a range of symptoms either not assessed by the FACT-G or else subsumed within its well-being scales. The FACT-G includes both symptoms and concerns within each scale [58]. The Medical Outcomes Study (MOS) [59], Sickness Impact Profile (SIP) [60], the SF-12 [61] and the M.D. Anderson Symptom Inventory (MDSAI) [62] were also used.

A variety of outcome measures were also used to assess sleep quality or insomnia. The Insomnia Severity Index (ISI) [63] was the most commonly used. Other measures included the Women's Health Initiative Insomnia Rating Scale (WHIIRS) [64] and the Pittsburgh Sleep Quality Index (PSQI) [65]. Broader QoL measures that assessed insomnia/sleep quality included the MDSAI [62] and the EORTC QLQ-C30 [56].

\section{Risk of bias assessment}

The included studies were assessed for risk of bias using the Cochrane 'Risk of Bias' Tool [44]. Some aspects of the studies were not reported with sufficient detail to assess bias and therefore were rated as unclear risk of bias for domains where insufficient information was provided. Further details are presented in Additional file 2.

\section{Random sequence generation (selection bias)}

Most studies described the process of allocating participants between study groups randomly, providing details about the method of randomisation employed. Eight 
studies did not describe random sequence generation in enough detail to allow a definite judgement.

In the majority of studies $(n=24)$, the method of allocation concealment either was not described or not described in sufficient detail to allow a definite judgement.

\section{Blinding (performance bias and detection bias)}

Most of the trials included in this review were at high risk of performance bias because, owing to the nature of the intervention, it was not possible to blind the trial personnel and participants. In a number of the studies were not described in sufficient detail to allow a definite judgement as to whether or not outcome assessors were blinded about the group allocation of participants.

\section{Incomplete outcome data (attrition bias)}

All studies provided some details of study attrition. Many of the studies $(n=19)$ were at a low risk of attrition bias, with good completion rates.

\section{Selective reporting (reporting bias)}

The majority of studies were at a low risk of reporting bias as, based on the information provided by the trial authors and study protocols (where available), it was unlikely that there was selective reporting of the primary and secondary outcomes. Sixteen of the trials were provided trial registration details.

\section{Other bias}

Most trials were deemed to be at a low risk for other biases such as potential bias due to baseline differences, inappropriate influence of the study sponsor and early stopping for benefit [12].

\section{Quality of the evidence}

We employed the GRADE approach to assess the evidence for the primary comparison of 'Psychological Interventions compared to usual care for Fatigue in cancer survivors'. As seen in Table 4, the majority of the evidence relating to psychological interventions for fatigue is of low quality, largely due to the finding that the available evidence is too heterogeneous to pool across studies. Further, it due to incomplete reporting of methods, it was difficult to ascertain risk of bias in studies. There is little evidence that directly answers the questions of interest for different types of psychological therapies.

\section{Effects of interventions}

In the published protocol, we had planned to conduct a meta-analysis, if it was deemed clinically meaningful and appropriate to do so [12]. However, given the heterogeneity in participant groups, study design, study comparators and measures used, we synthesised data narratively, as a meta-analysis would have been inappropriate.

\section{Comparison 1: psychological interventions (all types) vs. usual care}

Primary outcome: fatigue Eleven psychological interventions reported a significant effect of the intervention on an outcome of fatigue, compared to a waitlist control or usual care $[17,23,28,31,33,35,36,39$, $42,47,49]$.

\section{Secondary outcomes}

1. Global quality of life (QoL)/functional impact of fatigue

Global QoL/functional impact of fatigue was assessed in 19 of the 22 studies that compared a psychological intervention to a waitlist control or usual care. Thirteen of these 19 studies demonstrated a significant improvement compared to the control group, in at least one measure of QoL/functional impact of fatigue [22, 23, 25, 28, 30, $31,33,39,40,43,47-49]$. One study reported that participants assigned to the intervention group had significantly lower physical well-being compared to the control group at follow-up [21]. The remaining studies did not report any Group X Time interaction effects [15, 26, 34-36].

2. Fatigue self-efficacy Two studies assessed fatigue self-efficacy. Bower et al. [17] used the fatigue subscale of the HIV selfefficacy questionnaire and reported that Intervention group participants were significantly more confident than control group participants about their ability to manage fatigue and its impact on their lives at follow-up [17]. Foster et al. assessed fatigue using the Perceived Self-efficacy for Fatigue Self-management (PSEFSM). Initial evidence of improved fatigue self-efficacy at $\mathrm{T} 1$ in the intervention group was not maintained at final follow-up [26].

3. Mood

Mood was assessed over time in 18 of the 22 studies that compared a psychological intervention to a waitlist control or usual care. Ten of these reported significant improvements compared to the control group, in at least one measure of mood over time [21-23, 31, 35, 39, 43, 47, 49].

4. Sleep/insomnia

Sleep/ insomnia was assessed over time in 12 of the 22 studies that compared a psychological intervention to a waitlist control or usual care. Nine of these reported significant improvements compared to the control group, in at least one measure of sleep quality or insomnia symptoms over time [14, 16, 23, 31, 35, 36, 39, 47, 49]. Three of these studies were designed to specifically target 
Table 4 Grade evidence summary

\begin{tabular}{|c|c|c|}
\hline Outcomes & $\begin{array}{l}\text { № of } \\
\text { participants } \\
\text { (studies) }\end{array}$ & $\begin{array}{l}\text { Certainty of } \\
\text { the evidence }\end{array}$ \\
\hline
\end{tabular}

Psychological Interventions compared to usual care for Fatigue in cancer survivors

Follow up: range 2 weeks to 1 years $\quad 2918 \quad \oplus \oplus O$ LOW $^{\mathrm{a},}$ a. Downgraded $\mathrm{x} 1$ level for risk of bias due to all studies having high Intervention: Psychological Interventions (22 RCTs) b or unclear risk of performance bias. Many aspects of trial procedures Comparison: usual care were not reported in sufficient detail to adequately assess risk of bias in all domains of all included trials (e.g. unclear risk of selection bias in 18/ 22 studies, unclear risk of detection bias in 16/22).

b. Downgraded $x 1$ level for indirectness of evidence as many studies were combined interventions, which limit our ability to draw conclusions in relation to our research question relating solely to the effectiveness of psychological interventions. Generalizability of the findings are limited due to the high proportion of studies that recruited only/mostly breast cancer survivors. The majority of studies did not specifically target fatigue or screen for fatigue as part of inclusion criteria as recommended in existing guidelines. In some studies, it was difficult to assess when exactly participants completed cancer treatment prior to participating in the study. High levels of heterogeneity in sample and methods.

Subgroups of specific psychological intervention type (e.g. cognitive behavioural therapy) vs usual care

CBT interventions compared to usual care for 648(8 RCTs) Fatigue in cancer survivors

Follow up: range 1 months to 1 years

$\oplus \oplus O \mathrm{LOW}^{\mathrm{a}}$, a. Downgraded $\times 1$ level for risk of bias due to high/ unclear risk due to incomplete outcome data (attrition bias) in 5 of 8 studies Many aspects of trial procedures were not reported in sufficient detail to adequately assess risk of bias.

b. Downgraded $x 1$ level for indirectness of evidence as high levels of heterogeneity in sample and methods that limit the generalizability of the findings- While CBT was incorporated in all interventions to some degree, it was delivered in a variety of settings, modes and assessed in different ways. For example, $3 \times$ studies were not CBT interventions but were based on CBT strategies and 3x studies were focused specifically on CBT for insomnia.

Mindfulness-based interventions compared to usual care for Fatigue in cancer survivors Follow up: range 1 months to 4 months
749(6 RCTs) $\oplus \oplus 0 \mathrm{~L}$ LW ${ }^{\text {a, }}$ a. Downgraded $\times 1$ level for risk of bias due to high or unclear risk of performance bias in all studies. Many aspects of trial procedures were not reported in sufficient detail to adequately assess risk of bias. b. Downgraded $x 1$ level for indirectness of evidence as high levels of heterogeneity in sample and methods that limit the generalizability of the findings- While mindfulness was incorporated in all interventions to some degree, it was delivered in a variety of settings, modes and assessed in different ways.

1521 (8 RCTs) $\oplus \oplus \mathrm{OLOW}^{\mathrm{a}}$ a. a. Downgraded $\mathrm{x} 1$ level for risk of bias due to high or unclear risk of performance bias in all studies Some aspects of trial procedures were not reported in sufficient detail to adequately assess risk of bias b. Downgraded $x 1$ level for indirectness of evidence as high levels of heterogeneity - While all were psychological interventions, they were vastly different in sample and methods. Further, $4 \times$ studies were lifestyle interventions that incorporated other interventions such as physical activity and dietary changes.

GRADE Working Group grades of evidence

High certainty: We are very confident that the true effect lies close to that of the estimate of the effect

Moderate certainty: We are moderately confident in the effect estimate: The true effect is likely to be close to the estimate of the effect, but there is a possibility that it is substantially different

Low certainty: Our confidence in the effect estimate is limited: The true effect may be substantially different from the estimate of the effect

Very low certainty: We have very little confidence in the effect estimate: The true effect is likely to be substantially different from the estimate of effect

insomnia or sleep disturbance-all were effective for reducing fatigue $[23,36,39]$.

\section{Subgroup analysis and investigation of heterogeneity} In the original protocol, we specified that we would explore effects by subgroups of specific psychological intervention type (e.g. cognitive behavioural therapy) vs usual care.
Comparison 2: subgroups of specific psychological intervention type (e.g. cognitive behavioural therapy) vs. usual care

Cognitive-behavioural therapy vs. usual care Five studies reported on the effects of a CBT intervention compared to waitlist control or usual care $[23,28,33$, $36,39]$, of which three were focused specifically on CBT for insomnia (CBT-i) [23, 36, 39]. 
Primary outcome: fatigue Each of the five CBT studies reported significant effect of the intervention on fatigue over time [23, 28, 33, 36, 39]. Two other studies incorporated CBT strategies into the intervention. Dolbeault et al. [22] reported a significant effect on fatigue of a psycho-educational intervention based on CBT. Another study reported no significant differences between groups on a trial of Cognitively-Based Compassion Training [21]. Van der Lee et al. reported a significant effect of intervention over time using a combination of CBT and mindfulness strategies in a trial on mindfulness-based cognitive therapy [40].

\section{Secondary outcomes}

1. Global quality of life/functional impact of fatigue Four of the five CBT studies reported significant effect of the intervention over time at least one measure of Global QoL/functional impact of fatigue [23, 28, 33, 36, 39]. Savard et al. reported a significant group-time interaction global quality of life using the EORTC QLQ-C30 [39]. Using the Functional Assessment of Cancer Therapy Scalegeneral (FACT-G), Espie et al. [23] reported that CBT was associated with increased physical and functional QoL compared to the control group, at post-treatment and at follow-up. Using the SIP-8, both Prinsen et al. [33] Gielissen et al. [28] stated that the intervention condition reported a significantly greater decrease than patients in the waiting list condition in functional impairment. Ritterband et al. [36] reported that the group $\mathrm{x}$ time interaction for either the physical or mental subscale of the SF12 was not significant.

Using the EORTC core quality of life questionnaire (EORTC QLQ-C30), Dolbeault et al. reported greater improvement in emotional functioning, role functioning and global health status scales in the CBT-based psycho-educational intervention group compared with the control group. Group $\times$ time interaction effects were non-significant for the other subscales of the EORTC [22]. Using the SIP-8, van der Lee et al. reported that 6 months after the intervention, the mean well-being score at post measurement was significantly higher in the mindfulnessbased cognitive therapy intervention group than in the waiting list group corrected for pre-treatment level of well-being [40]. Conversely, participants assigned to cognitively-based compassion trainingh had significantly lower physical well-being compared to the control group at follow-up [21].

2. Fatigue self-efficacy

None of the five CBT studies assessed fatigue selfefficacy.

3. Mood
Mood was assessed over time in four of the five studies that compared a CBT intervention to a waitlist control or usual care [23, 28, 36, 39] -three of these reported a significant effect of the intervention on mood [23, 28, 39]. Gielissen et al. [28] assessed psychological distress using the Symptom Check List 90 and found that participants in the intervention condition reported a significantly greater decrease in psychological distress (95\% CI, 12.7 to $30.4, p<0.001)$ than patients in the waiting list condition. Using the Hospitals Anxiety and Depression Scale [HADS], Espie et al. [23] reported that CBT participants had reduced symptoms of anxiety, and depression relative to the control group (anxiety 95\% CI -0.92 to $-0.12, p=0.011$; depression $95 \% \mathrm{CI}-0.99$ to $0.19, p=0.004$ ). Also using the HADS, Savard et al. [39] reported significant group-time interactions on scores of anxiety $(p<.05)$ and depression $(p<.05)$. In contrast, Ritterband et al. [36] reported that the group $\times$ time interaction was not significant $(p=$ .09) on the total HADS score.

Dolbeault et al. [22] reported that a greater reduction of negative affect and improvement in positive affect was demonstrated in the intervention group compared with the control group. Significant group $\times$ time interactions indicated a positive effect of the intervention on anxiety, measured using the State-Trait Anxiety Inventory. Psychological adjustment-assessed with the Profile of Mood States (POMS) - demonstrated group $\times$ time interactions in favour of the intervention on anxiety, anger and depression. No effect of the intervention group was evidenced on The Mental Adjustment to Cancer Scale (MAC).

Dodds et al. [21] reported that compared to controls, at follow-up, participants assigned to the CBCT group demonstrated had significantly lower levels of perceived stress in the past week $(-1.6,95$ $\% \mathrm{CI}-3.1,-0.2)$-assessed using the Perceived Stress Scale (PSS-4). The Cognitive and Affective Mindfulness Scale-Revised (CAMS-R 10) demonstrated enhanced mindful presence in participants assigned to the CBCT group compared to controls, at follow-up $(3.1,95 \%$ CI 0.4, 5.8). There was no significant impact of the intervention on the other mood scales at final follow-up (week 12): Brief Center for Epidemiologic Studies-Depression questionnaire (CES-D-10), Fear of Cancer Recurrence Inventory (FCRI), the Impact of Events Scale-Revised (IES-R) or UCLA Loneliness Scale Version 3 (R-UCLA).

4. Sleep/insomnia Sleep/insomnia was assessed over time in four of the five studies that compared a CBT intervention 
to a waitlist control or usual car e[22, 23, 36, 39]three of these reported significant improvement compared to the control group, in at least one measure of sleep quality or insomnia symptoms over tim e[23, 36, 39].

Using the Insomnia Interview Schedule Insomnia Severity Index, Savard et al. [39] reported significant group-time interactions for all self-reported sleep variables, except for total sleep time. These included sleep efficiency, total wake time, sleep onset latency, wake after sleep onset.

Ritterband et al. [36] also employed the Insomnia Severity Index and reported a significant group $\times$ time interaction effect with the intervention group showing a significant improvement in insomnia severity from preto post-assessment, compared to the control group. These improvements were also clinically significant. Sleep Diary Variables were also used to assess sleep efficiency, sleep onset latency, wake after sleep onset and total sleep time. A significant group $\times$ time interaction was found for sleep efficiency and sleep onset latency with medium-to-large treatment effects $(d=.72$ and $d$ $=.67$ respectively). There was not a significant group $\mathrm{x}$ time interaction for wake after sleep onset, time in bed, number of awakenings or total sleep time. The intervention group also showed significantly more improvements than those in the control group on soundness of sleep and feeling restored, with large effect sizes (1.21 and 1.35 , respectively).

Espie et al. [23] also used sleep diaries to assess difficulty initiating (SOL) and maintaining (WASO) sleep.

Changes in total sleep time were not statistically significant, but improvements were seen in the CBT group WASO, SOL and Sleep efficiency scores. CBT was associated with median reduction in insomnia symptoms of almost $1 \mathrm{~h}(\mathrm{SOL}+\mathrm{WASO})$ compared with no change in the control group.

Dolbeault et al. [22] reported that no effect of the intervention group was evident over time, assessed using the EORTC QLQ-C30 sleep.

\section{Mindfulness-based interventions}

Six studies compared mindfulness-based interventions to waitlist control or usual care $[16,17,30,31,35,49]$. Two of the studies were specifically aimed at $\mathrm{CrF}[30$, $49]$ and another two were specifically focused on cancer $[16,35]$.

Primary outcome: fatigue Four of the studies on mindfulness-based interventions reported a significant effect of intervention on fatigue over time $[17,31,35$, 49]. One of the effective studies one was specifically aimed at $\mathrm{CrF}$ [49] and one was specifically focused on cancer [35]. The effective findings were not maintained at final follow up in one of the studies [17].

\section{Secondary outcomes}

1. Global quality of life/functional impact of fatigue Four of the mindfulness assessed Global QoL/ functional impact of fatigue [30, 31, 35, 49]. Three reported significant effect of the intervention over time on at least one measure of Global QoL /functional impact of fatigue [30, 31, 49]. Hoffman et al. [30] employed the breast-specific quality oflife scale FACT-B and the FACT-ES scale for endocrine symptoms and reported that mean scores in the intervention group were greater at both 8 and 12 weeks compared with the control group for all six measures (except social well-being which was significant at 8 weeks only). Using the WHO fiveitem well-being questionnaire (WHO-5), Hoffman et al. also reported significant increases in the intervention group compared with controls at both timepoints [30]. The authors also noted that increased hours of formal mindfulness classroom and home practice in the intervention group was associated with improved scores in FACT-ES, FACT-B, FACT physical well-being and WHO-5 at 12 weeks. Johns et al. assessed functional status using the Sheehan Disability Scale (SDS) and reported that the MBSR group demonstrated significantly lower functional disability scores than the control group at final follow-up with a large effect size $(d=-$ 1.22) [49]. Lengacher et al. used the M.D. Anderson Symptom Inventory (MDASI) [31]. They reported significant improvements in favour of MBSR(BC) in the symptom interference items (i.e., general activity, work (including work around the house) relations with other people, walking) and Housework, and Relationships. Using the Medical Outcomes Study Short-Form 36 (SF-36, v.2), Reich et al. [35] reported that group $\times$ time interaction was not significant for either mental or physical health.

2. Fatigue self-efficacy Bower et al. used the fatigue subscale of the HIV self-efficacy questionnaire and reported that Intervention group participants were significantly more confident than control group participants about their ability to manage fatigue and its impact on their lives at follow-up [17].

3. Mood

Mood was assessed over time in each of the six studies that compared mindfulness-based interventions to waitlist control or usual care $[16,17,30,31$, $35,49]$ - three of these reported a significant effect of the intervention on mood [31, 35, 49]. In the study by Reich et al. $[35,46]$, patients in the 
MBSR(BC) group showed significantly greater improvements in anxiety $(p=.007)$ assessed using the State-Trait Anxiety Inventory, and FORs (overall and problems; $p<.01$ ), as measured using the Concerns About Recurrence Scale. Results for depression (measured using CES-D) showed that participants assigned to MBSR $(\mathrm{BC})$ tended to report greater improvement than those in usual care; however, this trend did not reach statistical significance. The authors confirmed that improvement in both the cluster of psychological symptoms (anxiety, depression, perceived stress and quality of life (QOL), emotional well-being) ( $p=0.007)$ was related to assignment [35]. Lengacher et al. [31] assessed mood, enjoyment of life, distress and sadness, using the MDASI [62]. The MBSR(BC) intervention showed an improvement in mood, but not in distress or sadness. Johns et al. [49] assessed anxiety using the Patient Health Questionnaire Generalized Anxiety Disorder Scale-the MBSR group demonstrated significantly lower anxiety scores than the control group with a large effect size $(d=-0.98)$.Depression scores (measured using PHQ-8) were also significantly lower with large differences at final follow-up $(d=-1.71)$ [49]. Using the Beck Depression Inventory-II (BDI-II), Bower et al. [17] found that a significant Group $x$ time interaction at post-treatment was not maintained at 3 month follow-up. Stress decreased over the assessment period in both groups, as measured using the Perceived Stress Scale (PSS). Hoffman et al. [30] reported statistically significant improvements in outcome in the MBSR group compared with control group at both 8 and 12 weeks (for POMS total mood disturbance). The subscales of anxiety, depression showed these effects only at 8 week follow-up. Anger was significantly improved at 12 weeks but not at 8 weeks. The authors found that increased hours of formal mindfulness classroom and home practice in the MBSR group was associated with improved scores in POMS total mood disturbance [30]. Using the State Trait Anxiety ( STAI), Blaes et al. [16] found no significant difference between groups in anxiety despite a trend towards improvement for MBCR.

4. Sleep/insomnia

Sleep/insomnia was assessed over time in three studies that compared mindfulness-based interventions to waitlist control or usual care-two of these reported a significant effect of the intervention on sleep/insomnia over time $[16,49]$. Two of the studies assessed sleep quality using the Pittsburgh Sleep Quality Index (PSQI). Blaes et al. [16] reported that total sleep quality improved in those who received
MBCR compared to those in the control groupthis was maintained at 4 months. Conversely, Bower et al. [17] reported no significant effects for subjective sleep quality. Johns et al. [49] used the Insomnia Severity Index and reported that sleep disturbance was significantly improved for intervention group compared with the control condition at both follow-up points.

\section{Other psycho-social interventions vs. usual care}

The eight remaining interventions incorporated psychoeducation, motivational strategies and lifestyle and behaviour change approaches [14, 15, 25, 26, 43, 47, 51].

Primary outcome: fatigue A patient education program was reported to have improved fatigue [47], while a combined psycho-education and physical activity intervention showed that participants in the intervention group showed greater improvement in fatigue, but this was not a significant effect [25]. Health coaching was found to lead to a significant reduction on fatigue at 12 months but not at 3 months [42] and an intervention employing Motivational interviewing showed no significant differences between groups at 6 months [15]. Lifestyle interventions did reported mixed findings regarding their impact on fatigue, with one [14,34] reporting no significant differences between groups and one a significant effect of intervention at 6 months that was not maintained at 12 months [51]. An online intervention that aimed to enhance self-efficacy to manage problems associated with cancer-related fatigue following primary cancer treatment reported no significant changes in fatigue [26].

\section{Secondary outcomes}

1. Global quality of life /functional impact of fatigue Seven of the trials on other psycho-social interventions reported on Global QoL/functional impact of fatigue $[15,25,26,43,47,51]$. Four reported significant effect of the intervention over time on at least one measure of Global QoL/functional impact of fatigue $[25,43,47,48]$. Using the SF-36, Bennett et al. [15] noted that group $\times$ time interaction was not significant for either mental or physical health. Fillion et al. [28] reported marginal group $\times$ time interaction effects for physical quality of life in favour of the intervention group using the Medical Outcomes Study Short Form 12-Item Health Survey (SF-12). While mental quality of life showed no interaction or main effects, both conditions improved overtime. Conversely, there was no effect on the intervention on mental well-being. Three studies used the EORTC core quality of life questionnaire (EORTC QLQ-C30). In the 
study by Reif et al. [47], all functional and symptom scale values as well as single items values increased significantly in the intervention compared to the control group. Willems et al. also reported that the intervention was effective in increasing emotional and social functioning at 6 months [48]; however, these findings were not maintained at 12 months [51]. Similarly, Yun et al. [43] reported a significantly greater increase in global QOL and in emotional, cognitive and social functioning scores of EORTC QLQ-C30 scales. However, significance was lost on the emotional, and social functioning scores after Bonferroni corrections were applied for 15 multiple comparisons. Using the Functional Assessment of Cancer Therapy Scale-general (FACT-G), Foster et al. [26] did not report a significant effect of the intervention over time on the Fact-G measure.

2. Fatigue self-efficacy

Foster et al. did not reported improved fatigue selfefficacy at final follow-up [26].

3. Mood

Mood was assessed in six of the seven studies reporting on other psycho-social interventions [14, 25, 26, 43, 47, 51]. Yun et al. [43] reported that the web-based intervention group had clinically more meaningful improvement than the control group in HADS anxiety score. However, a statistically significant greater decrease in HADS was lost after Bonferroni corrections were applied. Willems et al. reported that another online intervention was effective in reducing HADS depression scores at 6months [48], but at 12 months from baseline, the intervention group no longer differed from the control group [51]. Reif et al. [47] also used the HADS and reported group $\times$ time interactions in favour of the intervention group for both anxiety and depression. Both Foster et al. [26] and Bantum et al. [14] reported a non-significant difference in groups in change over time using the Patient Health Questionnaire (PHQ-8). Fillion et al. [25] reported that no interaction effects for emotional distress (POMS anxiety + depression) were found.

\section{Sleep/insomnia}

Sleep/insomnia was assessed in three of the seven studies reporting on other psycho-social interventions [14, 43, 47]. Reif et al. reported an improvement in the intervention group, compared to the control group using the EORTC QLQ-C30 insomnia subscale [47]. Using the Women's Health Initiative Insomnia Rating Scale (WHIIRS), Bantum et al. [14] reported that the intervention group showed reduced insomnia from baseline to 6 months compared to the control group. Finally, Yun et al [43] did not report a significant effect of the intervention on scores on the Medical Outcome StudySleep Scale (MOS-SS) Sleep Quality Index I and II.

Further investigation of heterogeneity in trials comparing psychological interventions (all types) vs. usual care In the original protocol, we hypothesised that each of the factors below has the potential to have a clinically meaningful effect on the response to a psychological intervention amongst fatigued post-treatment cancer survivors.

1. Intervention for specific cancer type only vs intervention for any cancer type

2. In-person interventions vs remote interventions

3. Interventions specifically designed to treat fatigue after cancer treatment vs interventions not specific for fatigue

We performed narrative assessment of the influence of these factors on the primary outcomes. This narrative synthesis did not reveal any clear patterns in the findings based on differential influences of these factors on the effect of psychological interventions on fatigue.

\section{Comparison 3: intervention for specific cancer type only vs. intervention for any cancer type}

In a previous Cochrane review [8], it was noted that many of the studies of fatigued cancer patients during cancer included only breast cancer patients. Nine of the effective interventions in this review only included breast cancer patients. Seven studies that focused on breast cancer did not report a reduction in fatigue. Of the 17 studies with mixed samples, 13 reported a significant reduction in fatigue. However, breast cancer patients were often overrepresented in the studies of mixed samples. For example, one study [42] noted that over $60 \%$ of their sample had had breast cancer. Most studies included participants who had received a variety and combinations of cancer treatments (e.g. surgery, chemotherapy, radiotherapy). In one study [15], the authors specified that targeted patients were those who had received only radiotherapy.

\section{Comparison 4: in-person interventions vs. remote interventions}

Sixteen of the 22 trials compared that compared a psychological intervention to waitlist control or usual care were delivered in a group setting $[16,17,21-23,25,30$, $31,33,35,39,40,42,47,49$ ], with 11 of these reporting a reduction in fatigue over time $[17,22,23,31,33,35$, $39,40,42,47,49]$. The majority of the group interventions had 6-9 weekly 1-2.5 h sessions. Six included 
some homework or home practice $[17,21,30,31,35$, 49], with four of these studies reporting an effective reduction on fatigue $[17,31,35,49]$.

Two of the 22 trials that compared psychological interventions to waitlist control or usual care of the interventions involved individual face-to-face sessions-both of these were effective [28, 30]. One [42] of the two studies $[25,42]$ that offered telephone support were effective at reducing fatigue. A combination in-person/ telephone showed a reduction in fatigue at 3 months that was not maintained at 6 months [15]. Five of the studies reported on an online intervention [14, 26, 36, $43,51]$. The duration of these interventions varied from 6 weeks $[14,26,36]$ to 6 months [51]. All of the interventions were stand-alone interventions and two reported a significant reduction in fatigue at final followup $[36,43,51]$

\section{Comparison 5: interventions specifically designed to treat fatigue after cancer treatment vs. interventions not specific for fatigue}

This review sought to interventions that were specifically designed to treat fatigue after cancer treatment and interventions not specific for fatigue. Nine of the 22 trials that compared psychological interventions to waitlist control or usual care were interventions specific for fatigue $[16,25,26,28,33,40,43,47,49]$. Of the nine studies on interventions specific for fatigue, five assessed fatigue as part of inclusion criteria ([26, 40, 43, 47, 49]. Only one of these six studies did not report a significant effect on fatigue [26]. Two of the four studies interventions specific for fatigue that did not assess fatigue as part of inclusion criteria were effective [28, 33]. Three studies were specific interventions for insomnia or sleep disturbance-all were effective for reducing fatigue [23, 36, 39]. The remaining studies aimed to address lifestyle and quality of life or physical activity. Of these, six studies were effective in reducing fatigue $[15,17,22,31,42$, 51] at least one follow-up point. However, the effect of the intervention on fatigue was not maintained in two of these studies at final follow-up [15, 17, 22, 31, 42, 51].

\section{Discussion}

The aim of this review was to provide an overview of psychological interventions for fatigue after the completion of cancer treatment, and to evaluate the effectiveness of these interventions. In our search, 33 psychological interventions were identified, in which the effect on fatigue was tested in a RCT. The sample size of the included studies varied between 28 and 409, with 4525 participants overall. As with a previous review of interventions during treatment [8], the individual studies suggested that there is some evidence that psychological interventions are effective in reducing fatigue in cancer survivors. Twenty-three of the included studies reported a significant effect of the interventions on fatigue. However, the overall quality of the evidence about psychological interventions for fatigue after the completion of cancer treatment is low.

Given the heterogeneity in participant groups, study design, study comparators and measures used, we synthesised data narratively. Most interventions focused on psychoeducation, skills training, goal-setting, selfmonitoring, problem-solving, identification of maladaptive cognitions and emotion-focused coping strategies. Interventions also integrated behaviour therapy-oriented strategies including stimulus control and other techniques, targeting physical activity, sleep and stress management. However, studies differed widely in terms of mode, duration and frequency of the intervention delivery. This has also been reported in other reviews of nonpharmacological interventions for fatigue [66]. There were also differences in the extent of contact across the different interventions. It was not possible to establish if certain types of intervention were superior for reducing fatigue or if there was potentially an influence of heterogeneous specific disease sites and cancer treatments. These issues have previously been reported in other studies $[4,11,67]$.

Heterogeneity across the studies was also due to different definitions of fatigue criteria, various assessment tools and there were a number of different self-report measures used in the studies. As such, the same construct may not have been measured [68], as some tools were uni-dimensional, while others addressed the multidimensional nature of fatigue. Some of these measures were subscales of broader quality of life measures. Further, a number of these measures were designed specifically for cancer patients, while others were generic fatigue measures. Previous research has suggested that the lack of recommendations regarding fatigue measurement may be detrimental to research [68].

The strengths of this review includes the large number of studies included, a rigorous literature search based on a pre-published protocol; the use of independent raters; use of standard tools for reporting reviews and assessing bias in studies; and the presentation of a number of different variables that may be associated with intervention effectiveness. We are not aware of any studies that we have missed but acknowledge the potential for incomplete retrieval of identified research that may be a limitation of our review.

A number of limitations reduced our ability to make strong recommendations about any of the intervention strategies. In some studies, it was difficult to assess when exactly participants completed cancer treatment prior to participating in the study. As noted in similar reviews [68-70], the generalisability of the findings are limited due 
to the high proportion of studies that focused specifically on breast cancer or recruited a disproportionate number of breast cancer survivors. The majority of studies did not specifically target fatigue or screen for fatigue as part of inclusion criteria as recommended in existing guidelines $[1$, 6 , 66]. Few studies described the cancer treatment received by participants in detail, such as types of treatments and total duration. In terms of trial design, most studies did not report on the adherence of participants to the intervention treatment, adverse effects or integrity checks that may allow further inferences to be made about the quality of the studies. Blinding of participants is often not possible to achieve in studies of this nature. However, as noted in other reviews of fatigue [67], it is troublesome that a number of studies did not ensure blinding of outcome assessment given the subjective and self-reported nature of the outcomes. Many aspects of trial procedures were not reported in sufficient detail to adequately assess risk of bias in all domains of all included trials. Trials with negative results might not have been published at all, and therefore may have been missed during our search.

\section{Conclusion}

This review showed that there is some tentative support for psychological interventions for fatigue after cancer treatment based on the findings of individual studies. However, the RCTs were heterogeneous in nature and the number of high-quality studies was limited. Due to this heterogeneity, it is difficult to draw firm conclusions from the findings of this review. These findings demonstrate the need for the publication of more detailed descriptions of complex interventions, promoting methodological rigour and transparency in the design and throughout the trial process $[71,72]$. Future trials need to consider the multidimensional nature of $\mathrm{CrF}$ in order to improve our understanding of this complex symptom [67].

\section{Supplementary information}

Supplementary information accompanies this paper at https://doi.org/10. 1186/s13643-019-1230-2.

Additional file 1. Summary of Findings for Secondary outcomes.

Additional file 2. Risk of Bias Assessment.

Additional file 3. Search strategies used in this review.

\section{Abbreviations}

AMG: AnnMarie Groarke; ASCO: American Society of Clinical Oncology; BFI: Brief Fatigue Inventory; BMG: Brian E. McGuire; CIS: Checklist Individual Strength; CrF: Cancer-related fatigue; DD: Declan Devane; EC: Emma Carr; EORTC: European Organisation for Research and Treatment of Cancer; FACITF: Functional Assessment in Cancer Therapy - Fatigue; FACT: Functional Assessment of Cancer Therapy; FAQ: Fatigue Assessment Questionnaire; FSI: Fatigue Symptom Inventory; FSS: Fatigue Severity Scale; HADS: Hospital Anxiety and Depression Scale; IPOS: International Psycho-Oncology Society World Congress; ISI: Insomnia Severity Index; JW: Jane Walsh; MDSAI: M.D. Anderson Symptom Inventory; MeSH: Medical subject headings;
MFI: Multidimensional Fatigue Inventory; MFSI-SF: Multidimensional Fatigue Symptom Inventory-Short Form; MOS: Medical outcomes study;

NCCN: National Comprehensive Cancer Network; PHQ: Patient Health Questionnaire; PICO: Participants, Interventions, Comparisons, Outcome(s); POMS: The Profile of Mood States; PRISMA: Preferred Reporting Items for Systematic Reviews and Meta-Analyses; PSQI: Pittsburgh Sleep Quality Index; QOL: Quality of life; RCT: Randomised controlled trial; SF-12: Medical Outcomes Study Short Form 12-Item Health Survey; SIP: Sickness Impact Profile; STAI: State-Trait Anxiety Inventory; TC: Teresa Corbett; VAS: Visual analogue scale; WHIIRS: Women's Health Initiative Insomnia Rating Scale; WHO ICTRP: World Health Organization International Clinical Trials Registry Platform

\section{Acknowledgements}

Not applicable.

Declarations

Changes to the protocol

1. Secondary outcomes of interest to this review were specified a priori in the study protocol. However, in the review process, other frequently reported secondary outcomes were identified as relevant to this review. These outcomes of interest were Insomnia or sleep quality and pain. We have included these outcomes in the review.

2. In the published protocol, we had planned to conduct a metaanalysis, if it was deemed clinically meaningful and appropriate to do s o[12]. However, given the heterogeneity in participant groups, study design, study comparators and measures used, we synthesised data narratively, as a meta-analysis would have been inappropriate.

3. Due to this heterogeneity were also performed narrative assessment to explore effects by subgroups of specific psychological intervention type (e.g. cognitive behavioural therapy) vs. usual care.

4. Narrative assessment was also used to summarise the influence of these factors on the primary outcomes.

a. Intervention for specific cancer type only vs intervention for any cancer type

b. In-person interventions vs remote interventions

c. Interventions specifically designed to treat fatigue after cancer treatment vs interventions not specific for fatigue

5. A GRADE table has been added at the request of the editor

\section{Authors' contributions}

TC carried out initial background research and conceived of the study. TC also drafted the manuscript. EC, TC and BMG carried out screening process. $\mathrm{TC}$ and DD rated the quality of evidence using GRADE. BMG and DD have helped in drafting the manuscript or revising it critically for important intellectual content. AMG and JW have made substantial contributions to conception and design of the project, including revising the manuscript. All authors have given final approval of the version to be published.

\section{Funding}

The authors wish to acknowledge the funding provided by the Cancer Care West Hardiman Scholarship at the National University of Ireland Galway, Ireland. The funding body did not contribute to the design of the study and collection, analysis, and interpretation of data, or in writing the manuscript.

\section{Availability of data and materials}

The dataset supporting the conclusions of this article is included within the article (and its additional file(s)).

Ethics approval and consent to participate Not applicable.

Consent for publication

Not applicable.

Competing interests

The authors declare that they have no competing interests. 


\section{Author details}

${ }^{1} \mathrm{NIHR}$ ARC Wessex, School of Health Sciences, University of Southampton, Highfield, Southampton SO17 1BJ, UK. ${ }^{2}$ School of Psychology, National University of Ireland Galway, Galway, Ireland. ${ }^{3}$ School of Nursing and Midwifery, National University of Ireland Galway, Galway, Ireland.

Received: 11 February 2019 Accepted: 12 November 2019 Published online: 13 December 2019

\section{References}

1. Bower JE, et al. Screening, assessment, and management of fatigue in adult survivors of cancer: an American Society of Clinical oncology clinical practice guideline adaptation. J Clin Oncol. 2014;32(17):1840.

2. Berger AM, et al. Screening, evaluation, and management of cancer-related fatigue: Ready for implementation to practice? CA Cancer J Clin. 2015;65(3): 190-211.

3. Du S, et al. Patient education programs for cancer-related fatigue: a systematic review. Patient Educ Couns. 2015;98(11):1308-19.

4. O'Higgins C, et al. The pathophysiology of cancer-related fatigue: current controversies. Support Care Cancer. 2018;26:1-12.

5. Finnegan-John J, et al. A systematic review of complementary and alternative medicine interventions for the management of cancer-related fatigue. Integr Cancer Ther. 2013;12(4):276-90.

6. Mustian KM, et al. Comparison of pharmaceutical, psychological, and exercise treatments for cancer-related fatigue: a meta-analysis. JAMA Oncol. 2017;3(7):961-8.

7. Jacobsen PB, et al. Systematic review and meta-analysis of psychological and activity-based interventions for cancer-related fatigue. Health Psychol. 2007;26(6):660

8. Goedendorp, M.M., et al., Psychosocial interventions for reducing fatigue during cancer treatment in adults. The Cochrane Library, 2009.

9. Adam R, Bond C, Murchie P. Educational interventions for cancer pain. A systematic review of systematic reviews with nested narrative review of randomized controlled trials. Patient Educ Couns. 2015;98(3):269-82.

10. Newell SA, Sanson-Fisher RW, Savolainen NJ. Systematic review of psychological therapies for cancer patients: overview and recommendations for future research. J Natl Cancer Inst. 2002:94(8):558-84.

11. Minton $\mathrm{O}$, et al. Cancer-related fatigue and its impact on functioning. Cancer. 2013:119:2124-30.

12. Corbett T, et al. Protocol for a systematic review of psychological interventions for cancer-related fatigue in post-treatment cancer survivors. Syst Rev. 2015;4(1):174.

13. Moher D, et al. Preferred reporting items for systematic reviews and metaanalyses: the PRISMA statement. Ann Internal Med. 2009;151(4):264-9.

14. Bantum EOC, et al. Surviving and thriving with cancer using a Web-based health behavior change intervention: randomized controlled trial. J Med Internet Res. 2014;16(2):e52.

15. Bennett JA, et al. Motivational interviewing to increase physical activity in long-term cancer survivors: a randomized controlled trial. Nurs Res. 2007; 56(1):18-27.

16. Blaes AH, et al. Mindfulness-based cancer recovery in survivors recovering from chemotherapy and radiation. J Commun Support Oncol. 2016;14(8):351-8.

17. Bower JE, et al. Mindfulness meditation for younger breast cancer survivors: a randomized controlled trial. Cancer. 2015;121(8):1231-40.

18. Bruggeman-Everts FZ, et al. Effectiveness of two web-based interventions for chronic cancer-related fatigue compared to an active control condition: results of the 'Fitter na kanker' randomized controlled trial. J Med Internet Res. 2017;19(10):e336.

19. Carlson LE, et al. Randomized-controlled trial of mindfulness-based cancer recovery versus supportive expressive group therapy among distressed breast cancer survivors (MINDSET): long-term follow-up results. Psychooncology. 2016;25(7):750-9.

20. Dirksen SR, Epstein DR. Efficacy of an insomnia intervention on fatigue, mood and quality of life in breast cancer survivors. J Adv Nursing. 2008; 61(6):664-75.

21. Dodds SE, et al. Feasibility of Cognitively-Based Compassion Training (CBCT) for breast cancer survivors: a randomized, wait list controlled pilot study. Support Care Cancer. 2015;23(12):3599-608.

22. Dolbeault S, et al. The effectiveness of a psycho-educational group after early-stage breast cancer treatment: results of a randomized French study. Psychooncology. 2009;18(6):647-56.
23. Espie CA, et al. Randomized controlled clinical effectiveness trial of cognitive behavior therapy compared with treatment as usual for persistent insomnia in patients with cancer. J Clin Oncol. 2008;26(28):4651-8.

24. Ferguson RJ, et al. A randomized trial of videoconference-delivered cognitive behavioral therapy for survivors of breast cancer with selfreported cognitive dysfunction. Cancer. 2016;122(11):1782-91.

25. Fillion $L$, et al. A brief intervention for fatigue management in breast cancer survivors. Cancer Nursing. 2008;31(2):145-59.

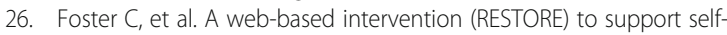
management of cancer-related fatigue following primary cancer treatment: a multi-centre proof of concept randomised controlled trial. Support Care Cancer. 2016;24(6):2445-53.

27. Freeman $L W$, et al. A randomized trial comparing live and telemedicine deliveries of an imagery-based behavioral intervention for breast cancer survivors: reducing symptoms and barriers to care. Psychooncology. 2015; 24(8):910-8

28. Gielissen MF, et al. Effects of cognitive behavior therapy in severely fatigued disease-free cancer patients compared with patients waiting for cognitive behavior therapy: a randomized controlled trial. J Clin Oncol. 2006;24(30):4882-7.

29. Heckler CE, et al. Cognitive behavioral therapy for insomnia, but not armodafinil, improves fatigue in cancer survivors with insomnia: a randomized placebo-controlled trial. Support Care Cancer. 2016;24(5):2059-66.

30. Hoffman CJ, et al. Effectiveness of mindfulness-based stress reduction in mood, breast-and endocrine-related quality of life, and well-being in stage 0 to III breast cancer: a randomized, controlled trial. J Clin Oncol. 2012; 30(12):1335-42.

31. Lengacher CA, et al. Mindfulness based stress reduction in post-treatment breast cancer patients: an examination of symptoms and symptom clusters. Journal of behavioral medicine. 2012;35(1):86-94.

32. Matthews EE, et al. Cognitive behavioral therapy for insomnia outcomes in women after primary breast cancer treatment: a randomized, controlled trial. Oncol Nurs Forum. 2014;41(3):241-53.

33. Prinsen $\mathrm{H}$, et al. The role of physical activity and physical fitness in postcancer fatigue: a randomized controlled trial. Support Care Cancer. 2013;21(8):2279-88.

34. Reeves M, et al. The Living Well after Breast Cancer ${ }^{\mathrm{TM}}$ Pilot Trial: A weight loss intervention for women following treatment for breast cancer. Asia Pac J Clin Oncol. 2017;13(3):125-36.

35. Reich RR, et al. Mindfulness-based stress reduction in post-treatment breast cancer patients: immediate and sustained effects across multiple symptom clusters. J Pain Symptom Manage. 2017;53(1):85-95.

36. Ritterband $L M$, et al. Initial evaluation of an Internet intervention to improve the sleep of cancer survivors with insomnia. Psychooncology. 2012;21(7): 695-705.

37. Rogers $L Q$, et al. Effects of a multicomponent physical activity behavior change intervention on fatigue, anxiety, and depressive symptomatology in breast cancer survivors: randomized trial. Psychooncology. 2017;26(11):1901-6.

38. Sandler CX, et al. Randomized evaluation of cognitive-behavioral therapy and graded exercise therapy for post-cancer fatigue. J Pain Symptom Manage. 2017:54(1):74-84

39. Savard J, et al. Randomized study on the efficacy of cognitive-behavioral therapy for insomnia secondary to breast cancer, part I: Sleep and psychological effects. J Clin Oncol. 2005;23(25):6083-96.

40. van der Lee ML, Garssen B. Mindfulness-based cognitive therapy reduces chronic cancer-related fatigue: a treatment study. Psychooncology. 2012; 21(3):264-72.

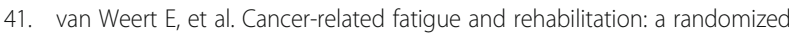
controlled multicenter trial comparing physical training combined with cognitive-behavioral therapy with physical training only and with no intervention. Phys Ther. 2010;90(10):1413-25.

42. Yun $\mathrm{YH}$, et al. A randomized controlled trial of physical activity, dietary habit, and distress management with the Leadership and Coaching for Health (LEACH) program for disease-free cancer survivors. BMC cancer. 2017; 17(1):298

43. Yun $\mathrm{YH}$, et al. Web-based tailored education program for disease-free cancer survivors with cancer-related fatigue: a randomized controlled trial. J Clin Oncol. 2012;30(12):1296-303.

44. Higgins J, Green S. Cochrane handbook for systematic reviews of interventions Version 5.1. 0. The Cochrane Collaboration. Confidence intervals; 2011. 
45. Carlson LE, et al. Randomized controlled trial of mindfulness-based cancer recovery versus supportive expressive group therapy for distressed survivors of breast cancer. J Clin Oncol. 2013;31(25):3119-26.

46. Lengacher $\mathrm{CA}$, et al. Examination of broad symptom improvement resulting from mindfulness-based stress reduction in breast cancer survivors: a randomized controlled trial. J Clin Oncol. 2016:34(24):2827.

47. Reif $K$, et al. A patient education program is effective in reducing cancerrelated fatigue: a multi-centre randomised two-group waiting-list controlled intervention trial. Eur J Oncol Nursing. 2013;17(2):204-13.

48. Willems RA, et al. Short-term effectiveness of a web-based tailored intervention for cancer survivors on quality of life, anxiety, depression, and fatigue: randomized controlled trial. Psychooncology. 2017;26(2):222-30.

49. Johns SA, et al. Randomized controlled pilot study of mindfulness-based stress reduction for persistently fatigued cancer survivors. Psychooncology. 2015;24(8):885-93.

50. Rogers $L Q$, et al. Physical activity and health outcomes three months after completing a physical activity behavior change intervention: persistent and delayed effects. Cancer Epidemiol Biomarkers Prev. 2009;18(5):1410-8.

51. Willems RA, et al. Long-term effectiveness and moderators of a web-based tailored intervention for cancer survivors on social and emotional functioning, depression, and fatigue: randomized controlled trial. J Cancer Survivorship. 2017;11(6):691-703.

52. Zigmond AS, Snaith RP. The hospital anxiety and depression scale. Acta Psychiatrica Scandinavica. 1983;67(6):361-70.

53. Spitzer RL, Williams JB, Kroenke K; Patient Health Questionnaire Primary Care Study Group. Validation and utility of a self-report version of PRIME-MD: the PHQ primary care study. JAMA. 1999;282(18):1737-44.

54. Lorr M, McNair DM. Profile of mood states-bipolar form. San Diego: Educational and Industrial Testing Service; 1988.

55. Spielberger CD, et al. State-trait anxiety inventory (STAI). BiB, 2010; 1970. p. 180

56. Aaronson NK, et al. The European Organization for Research and Treatment of Cancer QLQ-C30: a quality-of-life instrument for use in international clinical trials in oncology. JNCl: J Natl Cancer Institute. 1993;85(5):365-76.

57. Cella DF, et al. The Functional Assessment of Cancer Therapy scale: development and validation of the general measure. J Clin Oncol. 1993; 11(3):570-9.

58. Luckett T, et al. Choosing between the EORTC QLQ-C30 and FACT-G for measuring health-related quality of life in cancer clinical research: issues, evidence and recommendations. Ann Oncol. 2011;22(10):2179-90.

59. Stewart AL, Hays RD, Ware JE. The MOS short-form general health survey: reliability and validity in a patient population. Med Care. 1988;26(7):724-35.

60. Bergner $\mathrm{M}$, et al. The Sickness Impact Profile: development and final revision of a health status measure. Med Care. 1981;19:787-805.

61. Ware JE Jr, Kosinski M, Keller SD. A 12-Item Short-Form Health Survey: construction of scales and preliminary tests of reliability and validity. Med Care. 1996;34(3):220-33.

62. Cleeland CS, et al. Assessing symptom distress in cancer patients: the MD Anderson Symptom Inventory. Cancer. 2000;89(7):1634-46.

63. Morin CM. Insomnia: Psychological assessment and management. New York: Guilford Press; 1993.

64. Levine DW, et al. Reliability and validity of Women's Health Initiative Insomnia Rating Scale. Psycholog Assess. 2003;15(2):137.

65. Buysse DJ, et al. The Pittsburgh Sleep Quality Index: a new instrument for psychiatric practice and research. Psychiatry Res. 1989;28(2):193-213.

66. Hulme $\mathrm{K}$, et al. Fatigue interventions in long term, physical health conditions: A scoping review of systematic reviews. PloS one. 2018;13(10):e0203367.

67. Bennett $\mathrm{S}$, et al. Educational interventions for the management of cancerrelated fatigue in adults. Cochrane Database Syst Rev. 2016;11:CD008144.

68. Pearson $\mathrm{E}_{\text {, et }}$ al. Interventions for cancer-related fatigue: a scoping review. Eur J Cancer Care. 2018;27(1):e12516.

69. Kelley GA, Kelley KS. Exercise and cancer-related fatigue in adults: a systematic review of previous systematic reviews with meta-analyses. BMC Cancer. 2017;17(1):693.

70. Johnson JA, et al. A systematic review and meta-analysis of randomized controlled trials of cognitive behavior therapy for insomnia (CBT-I) in cancer survivors. Sleep Med Rev. 2016;27:20-8.

71. Craig P, et al. Developing and evaluating complex interventions: the new Medical Research Council guidance. Int J Nursing Stud. 2013;50(5):587-92.

72. Hoffmann TC, et al. Better reporting of interventions: template for intervention description and replication (TIDieR) checklist and guide. BMJ. 2014;348:g1687.

\section{Publisher's Note}

Springer Nature remains neutral with regard to jurisdictional claims in published maps and institutional affiliations.
Ready to submit your research? Choose BMC and benefit from:

- fast, convenient online submission

- thorough peer review by experienced researchers in your field

- rapid publication on acceptance

- support for research data, including large and complex data types

- gold Open Access which fosters wider collaboration and increased citations

- maximum visibility for your research: over $100 \mathrm{M}$ website views per year

At BMC, research is always in progress.

Learn more biomedcentral.com/submissions 